\title{
Partitions of large Rado graphs
}

\author{
M. Džamonja* \\ School of Mathematics \\ University of East Anglia \\ Norwich, NR4 7TJ, UK \\ J.A. Larson* and W.J. Mitchell* \\ Department of Mathematics \\ University of Florida-Gainesville \\ 358 Little Hall, PO Box 118105 \\ Gainesville, FL 32611-8105, USA
}

July 22, 2008

\begin{abstract}
Let $\kappa$ be a cardinal which is measurable after generically adding $\beth_{\kappa+\omega}$ many Cohen subsets to $\kappa$ and let $\mathcal{G}=(\kappa, E)$ be the $\kappa$-Rado graph. We prove, for $2 \leq m<\omega$, that there is a finite value $r_{m}^{+}$such that the set $[\kappa]^{m}$ can be partitioned into classes $\left\langle C_{i}: i<r_{m}^{+}\right\rangle$such that for any coloring of any of the classes $C_{i}$ in fewer than $\kappa$ colors, there is a copy $\mathcal{G}^{*}$ of $\mathcal{G}$ in $\mathcal{G}$ such that $\left[\mathcal{G}^{*}\right]^{m} \cap C_{i}$ is monochromatic. It follows that $\mathcal{G} \rightarrow(\mathcal{G})_{<\kappa / r_{m}^{+}}^{m}$, that is, for any coloring of $[\mathcal{G}]^{m}$ with fewer than $\kappa$ colors there is a copy $\mathcal{G}^{\prime}$ of $\mathcal{G}$ such that $\left[\mathcal{G}^{\prime}\right]^{m}$ has at most $r_{m}^{+}$colors. On the other hand, we show that there are colorings of $\mathcal{G}$ such that if $\mathcal{G}^{\prime}$ is any copy of $\mathcal{G}$ then $C_{i} \cap\left[\mathcal{G}^{\prime}\right]^{m} \neq \emptyset$ for all $i<r_{m}^{+}$, and hence $\mathcal{G} \nrightarrow[\mathcal{G}]_{r_{m}^{+}}^{m}$.

We characterize $r_{m}^{+}$as the cardinality of a certain finite set of types and obtain an upper and a lower bound on its value. In particular, $r_{2}^{+}=2$ and for $m>2$ we have $r_{m}^{+}>r_{m}$ where $r_{m}$ is the corresponding number of types for the countable Rado graph.
\end{abstract}

\section{Introduction}

The random or Rado graph is the unique countable graph $G$ which has the property that for all finite disjoint sets $A$ and $B$ of vertices in $G$ there is a

\footnotetext{
* Research of the first and second authors was partially supported by Engineering and Physical Sciences Research Council and research of the third author was partly supported by grant number DMS 0400954 from the United States National Science Foundation.

The second and third authors thank the University of East Anglia and the University of Münster for their gracious hospitality.

AMS Mathematics Subject Classification (2000): MSC 03E02, MSC 05D10
} 
vertex $c$ such that there is an edge from $c$ to each element of $A$ and to none of the elements of $B$. If $\kappa$ is any cardinal satisfying $\kappa^{<\kappa}=\kappa$, one can similarly define the $\kappa$-Rado graph $\mathcal{G}_{\kappa}$ whose set of vertices is of size $\kappa$ and which satisfies that for all disjoint sets $A$ and $B$ of vertices, each of size $<\kappa$, there is a vertex $c$ such that there is an edge from $c$ to each element of $A$ and to none of the elements of $B$. Graph $\mathcal{G}_{\kappa}$ is unique up to isomorphism. Partition properties of the random graph were studied by several authors, one can see a detailed discussion in J. Larson's paper [5]. Using a technique we introduced in our paper [1] along with a fine analysis of the types of finite subsets of $\mathcal{G}_{\kappa}$ we are able to prove the following partition theorem. By $\mathbb{G}$ we mean a fixed copy of $\mathcal{G}_{\kappa}$ whose universe is $\kappa$.

Theorem 1.1. Let $m \geq 2$ and suppose that $\kappa$ is a cardinal which is measurable in the generic extension obtained by adding $\lambda$ Cohen subsets of $\kappa$, where $\lambda \rightarrow$ $(\kappa)_{2^{\kappa}}^{2 m}$. Then for $r_{m}^{+}$equal to the number of vip $m$-types, any $\kappa$-Rado graph $\mathbb{G}=(\kappa, E)$ satisfies

$$
\mathbb{G} \rightarrow(\mathbb{G})_{<\kappa, r_{m}^{+}}^{m} \text { and } \mathbb{G} \nrightarrow(\mathbb{G})_{<\kappa, r_{m}^{+}-1}^{m} .
$$

Moreover, there is a canonical partition of $[\mathbb{G}]^{m}$ into $r_{m}^{+}$parts.

We have used the following definition

Definition 1.2. A partition $\mathcal{C}=\left\{C_{0}, C_{1}, \ldots, C_{r}\right\}$ of $[\mathbb{G}]^{m}$ is a canonical partition if it is persistent and indivisible. To be persistent means that if $\mathbb{G}^{*}$ is any copy of $\mathbb{G}$ within $\mathbb{G}$ then $\left[\mathbb{G}^{*}\right]^{m} \cap C_{j} \neq \emptyset$ for each $j<r$. To be indivisible means that for every coloring of $\mathbb{G}$ there is a copy $\mathbb{G}^{*}$ of $\mathbb{G}$ within $\mathbb{G}$ such that $\left[\mathbb{G}^{*}\right]^{m} \cap C_{j}$ is monochromatic for each $j<r$.

Results analogous to this theorem were already known for $\kappa=\omega$ through the work of Laflamme, Sauer and Vuksanovic [3]. Many historical points and a discussion of the use and to a certain extent necessity of the large cardinal assumptions we have are given in [1].

In [1] we obtained a partition theorem for $\kappa$-dense linear orders whose statement is analogous to the statement of our Theorem 1.1. The relevant types are much more complicated for graphs than for orders and to capture them we need a better Diagonalization Lemma than the one provided in [1]; this is achieved in Lemma 3.3. Also we must work harder to show that the relevant types are realized by the ranges of the maps we use to build our copies of the Rado graph inside itself; this is achieved in Corollary 5.7.

The paper is organized as follows: in $\S 2$ we embed the $\kappa$-Rado graph in a complete binary tree of height $\kappa$ and translate the notion of a copy of the $\mathbb{G}$ inside $\mathbb{G}$ into the new setting. We also introduce notation and several definitions related to trees and embeddings of their subsets, and prove some basic theorems needed for the rest of the paper. In $\S 3$ we prove the existence of certain nice mappings of the complete binary tree of height $\kappa$ into itself, which are used in the definition of the canonical partition and in the proof of its indivisibility which gives the existence of the numbers $r_{m}^{+}$. In $\S 4$, we show that all the relevant 
types are realized by subsets of the the ranges of the nice mappings defined in $\S 3$. In $\S 5$ we group lemmas necessary for the proof of the persistence, in particular showing that all the relevant types are realized by subsets of the the ranges of a wider class of mappings and prove a Uniformization Theorem. Finally in $\S 6$ we use the Uniformization Theorem to prove the main theorem and give some ideas of how large $r_{m}^{+}$is for some small values of $m$.

For the remainder of the paper an unattributed $m$ will mean a natural number with $2 \leq m$ and $\kappa$ a cardinal satisfying the hypotheses of the main theorem for some number $m$. In particular, $\kappa=\kappa^{<\kappa}$ and $\kappa$ is a strong limit. For any cardinal $\lambda$, let $[A]^{\lambda}$ denote the collection of all subsets of $A$ of cardinality $\lambda$, and let $[A]^{<\lambda}$ denote the collection of all subsets of $A$ of cardinality less than $\lambda$. We write $\mathbb{G}=(\kappa, E)$ for the $\kappa$-Rado graph $\mathcal{G}_{\kappa}$.

\section{Translation}

In this section we give an embedding of the $\kappa$-Rado $\mathbb{G}$ graph into the complete rooted binary tree of height $\kappa$, prove a Translation Theorem matching increasing isomorphisms of $\mathbb{G}$ with pnp maps, defined below. We also define strong embeddings and $m$-types prove a preservation property of strong embeddings.

The first lemma of the section states that any induced subgraph of a $\kappa$-Rado graph has an induced subgraph which is isomorphic to the $\kappa$-Rado graph by an isomorphism that also preserves $<$.

Lemma 2.1. For any $H \subseteq \kappa$ with $\mathbb{G} \cong(H, E\lceil H)$ there is a $<$-increasing map $g: \kappa \rightarrow H$ with $\mathbb{G} \cong(g[\kappa], E\lceil g[\kappa])$.

Proof. Fix attention on a specific induced subgraph $(H, E\lceil H)$ isomorphic to $\mathbb{G}$. Let $h: \kappa \rightarrow H$ be the isomorphism.

By the definition of the $\kappa$-Rado graph for any $\gamma<\kappa$ and any subset $A \subseteq \gamma$, there are cofinally many $\zeta<\kappa$ with $\{\delta<\gamma:\{\delta, \zeta\} \in E\}=A$.

Define $z: \kappa \rightarrow \kappa$ and $g: \kappa \rightarrow H$ by recursion. Let $z(0)=0$ and $g(0)=$ $h(0)$. Suppose $z\lceil\alpha$ and $g\lceil\alpha$ have been defined so that $z$ is increasing, for all $\beta<\alpha, g(\beta)=h(z(\beta))$, and $g\lceil\alpha$ is an increasing endomorphism of $(\alpha, E\lceil\alpha)$ into $(H, E\lceil H)$. Let $\gamma>\alpha$ be so large that if $h(\eta)<\sup \{g(\beta)+1: \beta<\alpha\}$, then $\gamma>\eta$. Let $A_{\alpha}:=\{z(\beta): \beta<\alpha \wedge\{\beta, \alpha\} \in E\}$. Let $\zeta=z(\alpha) \geq \gamma$ be such that $\{\delta<\gamma:\{\delta, \zeta\} \in E\}=A_{\alpha}$. Let $g(\alpha)=h(z(\alpha))$. Since $h$ is an isomorphism, a pair $\{h(z(\beta)), h(z(\alpha))\}$ is in $E$ if and only if the pair $\{z(\beta), z(\alpha)\}$ is in $E$. It follows that $\{\beta<\alpha:\{g(\beta), g(\alpha)\} \in E\}=A_{\alpha}$. Therefore by induction, $g$ is the desired endomorphism into $H$.

In all the future instances we will assume that any copy of $\mathbb{G}$ inside $\mathbb{G}$ is obtained using an increasing isomorphism.

The next step in our proof is to embed the $\kappa$-Rado graph into a tree. For an infinite cardinal $\kappa$, the set ${ }^{\kappa>} 2$, ordered by end-extension, $\subseteq$, is the complete binary tree on $\kappa$ with root the empty sequence, $\emptyset$. For our embedding, we generalize the approach used by Erdős, Hajnal and Pósa [2]. 
Definition 2.2. The tree embedding of $\mathbb{G}$ into ${ }^{\kappa>} 2$ is the function $\sigma: \kappa \rightarrow^{\kappa>} 2$ defined by $\sigma(0)=\emptyset$, and for $\alpha>0, \sigma(\alpha): \alpha \rightarrow 2$ is defined by $\sigma(\alpha)(\beta)=1$ if and only if $\{\alpha, \beta\} \in E$. Let $S^{*}=\sigma[\kappa]$.

Note that $\sigma$ is necessarily injective and that for any $\alpha<\kappa, \lg (\sigma(\alpha))=\alpha$. We next introduce two properties of the range of the embedding $\sigma$.

Definition 2.3. A subset $S \subseteq{ }^{\kappa>} 2$ is cofinal above $w$ if for all $t \in \operatorname{Cone}(w)$ there is some $s \in S$ with $t \subseteq s$. If $w=\emptyset$, we say $S$ is cofinal.

For any tree $T \subseteq{ }^{\kappa>} 2$, a subset $S \subseteq T$ is transverse if it has at most one node on each level of $T$.

Lemma 2.4. $S^{*}$ is a cofinal transverse subset of ${ }^{\kappa>} 2$.

Proof. By definition of $\sigma$, for all $\alpha<\kappa, \lg (\sigma(\alpha))=\alpha$, so $S^{*}$ is transverse.

To see that $S^{*}$ is cofinal, suppose $s \in{ }^{\kappa>} 2$. Let $\alpha=\lg (s)$ and let $A$ be the set of all $\beta<\lg (s)$ with $s(\beta)=1$. Since $\mathbb{G}$ is a $\kappa$-Rado graph, there is an element $\gamma>\alpha$ such that $\{\beta, \gamma\} \in E$ for all $\beta \in A$ and $\{\beta, \gamma\} \notin E$ for all $\beta \in \alpha \backslash A$. It follows that $s \subseteq \sigma(\gamma)$. Thus $\sigma[\kappa]$ is cofinal in ${ }^{\kappa>} 2$.

We review terminology related to trees of large height.

Definition 2.5. For any tree $T$ of sequences ordered by end extension, and any ordinal $\alpha$, the $\alpha$ th level of $T$, in symbols $T(\alpha)$, is the set of all nodes $t \in T$ for which $\alpha$ is the order type of the set of predecessors of $t$, namely $\{s \in T: s \subsetneq t\}$. For $s \in T$ the length $\lg (s)$ is defined to be $\alpha$ if and only if $s \in T(\alpha)$.

Our next goal is a translation of questions about isomorphic copies of the $\kappa$ Rado graphs in itself to questions about ${ }^{\kappa>} 2$. Toward that end, we define passing number preserving maps. This notion was used in the proof of the limitation of colors result by Laflamme, Sauer and Vuksanovic [3] for the countable Rado graph.

Definition 2.6. For $s, t \in{ }^{\kappa>} 2$ with $\lg (t)>\lg (s)$, call $t(\lg (s))$ the passing number of $t$ at $s$. Call a function $f:{ }^{\kappa>} 2 \rightarrow{ }^{\kappa>} 2$ passing number preserving or a pnp map if it preserves

1. length order: $\lg (s)<\lg (t)$ implies $\lg (f(s))<\lg (f(t))$; and

2. passing numbers: $\lg (s)<\lg (t)$ implies $f(t)(\lg (f(s)))=t(\lg (s))$.

Lemma 2.7. For any <-increasing map $g: \kappa \rightarrow \kappa$ with $\mathbb{G} \cong(g[\kappa], E\lceil g[\kappa])$, the composition $\sigma \circ g \circ \sigma^{-1}: S^{*} \rightarrow S^{*}$ is a pnp map.

Proof. Let $f:=\sigma \circ g \circ \sigma^{-1}$ for some <-increasing endomorphism $g$ of $\mathbb{G}$ into itself. Suppose $s, t \in S^{*}$ and $\beta:=\lg (s)<\lg (t)=\alpha$. Then $\sigma^{-1}(s)=\beta$ and $\sigma^{-1}(t)=\alpha$. Since $g$ is <-increasing, $g(\beta)<g(\alpha)$. Hence $\lg (f(s))=g(\beta)<$ $g(\alpha)=\lg (f(t))$. Moreover, $t(\lg (s))=t(\beta)=1$ if and only if $\{\beta, \alpha\} \in E$. Since $g$ is an endomorphism, $t(\lg (s))=1$ if and only if $\{g(\beta), g(\alpha)\} \in E$. By definition of the tree embedding, it follows that $t(\lg (s))=1$ if and only if $f(t)(\lg (s))=1$. Thus $f$ is a pnp map from $S^{*}$ into $S^{*}$. 
By much the same reasoning, one can show the converse.

Theorem 2.8. [Translation Theorem] For any pnp map $f: S^{*} \rightarrow S^{*}$, the composition $g:=\sigma^{-1} \circ f \circ \sigma: \kappa \rightarrow \kappa$ is an <-increasing map with $\mathbb{G} \cong(g[\kappa], E\lceil g[\kappa])$.

Proof. Let $g:=\sigma^{-1} \circ f \circ \sigma$ for some pnp map $f: S \rightarrow S$. Suppose $\beta<\alpha<\kappa$. Then $\lg (\sigma(\beta))=\beta<\alpha=\lg (\sigma(\alpha))$. Since $f$ is a pnp map, $\sigma^{-1} \circ f \circ \sigma(\beta)=$ $\lg (f(\sigma(\beta)))<\lg (f(\sigma(\alpha)))=\sigma^{-1} \circ f \circ \sigma(\alpha)$, so $g$ is a $<$-increasing map.

By the definition of $\sigma,\{\beta, \alpha\}$ is an edge of $\mathbb{G}$ if and only if $\sigma(\alpha)(\lg (\sigma(\beta)))=$ 1. Since $f$ is a pnp map, it follows that $\{\beta, \alpha\}$ is an edge of $\mathbb{G}$ if and only if $f(\sigma(\alpha))(\lg (f(\sigma(\beta))))=1$. Apply the definition of $\sigma$ to the pair $g(\alpha)=$ $\sigma^{-1}(f(\sigma(\alpha)))$ and $g(\beta)=\sigma^{-1}(f(\sigma(\beta)))$, to see that $\{\beta, \alpha\}$ is an edge of $\mathbb{G}$ if and only if $\{g(\beta), g(\alpha)\}$ is an edge.

Thus $g$ is a $<$-increasing endomorphism of $\mathbb{G}$ into itself.

Definition 2.9. For any subsets $S_{0}$ and $S_{1}$ of ${ }^{\kappa>} 2$, a function $e: S_{0} \rightarrow S_{1}$ is a strong embedding if it is an injection with the following preservation properties:

1. (extension) $s \subseteq t$ if and only if $e(s) \subseteq e(t)$;

2. (length order) $\lg (s)<\lg (t)$ if and only if $\lg (e(s))<\lg (e(t))$ and $\lg (s)=$ $\lg (t)$ if and only if $\lg (e(s))=\lg (e(t))$;

3. (passing number) if $\lg (s)<\lg (t)$, then $e(t)(\lg (e(s)))=t(\lg (s))$.

Lemma 1.6. from [1] shows that being the range of a strong embedding is equivalent to being a strongly embedded subset which has another, somewhat more combinatorial definition.

We review additional definitions and notation in preparation for the definition of strongly diagonal set which is critical to our isolation of the types underlying the canonical partition. Call two elements $s$ and $t$ of ${ }^{\kappa>} 2$ incomparable if neither is an end-extension of the other. A subset $A$ of $T$ is an antichain if all $s \neq t$ are incomparable. By $s \wedge t$ denote the meet of $s$ and $t$, namely the longest initial segment of both $s$ and $t$. For any subset $S \subseteq T$, let $S^{\wedge}$ denote the meet closure of $S$, i.e. the set $\{u: u=s \wedge t$ for some $s, t \in S\}$.

Definition 2.10. A set $A \subseteq{ }^{\kappa>} 2$ is diagonal if it is an antichain, and its meet closure, $A^{\wedge}$, is transverse. It is called strongly diagonal if, in addition, for all $t \in A$ and all $s \in A^{\wedge} \backslash\{t\}$, the following implication holds:

$(\lg (s)<\lg (t) \wedge t(\lg (s))=1) \Longrightarrow s \subseteq t$ or $s$ has no proper extension in $A$.

Recall that for any tree $T=(T, \subseteq)$, a node $s$ is a leaf of $T$ or terminal node of $T$ if for all $t \in T \backslash\{s\}$, one has $s \nsubseteq t$.

Definition 2.11. A similarity tree is a finite subtree of ${ }^{\omega>} 2$ closed under initial segments and such that every level contains at least one leaf node or meet of leaf nodes. Call $\tau$ an $m$-type if it is a downward closed subtree of ${ }^{2 m-2 \geq} 2$ whose set $L$ of leaves is an $m$-element strongly diagonal set. 
The $m$-types, defined above, can be used to define an equivalence relation on $m$-element subsets of a strongly diagonal set where two such anti-chains are equivalent if their collapses, defined below, are the same $m$-type. At a later point we will add order to get the vip $m$-types that are used in the definition of the canonical partition.

Definition 2.12. If $x$ is a finite subset of ${ }^{\kappa>} 2$ then we write $\operatorname{clp}(x)$ for the collapse of $x$, which is the subtree $y$ of ${ }^{\omega>} 2$ that includes the root and is of minimal possible height such that there is a strong embedding from $x^{\wedge}$ onto the closure $z^{\wedge}$ of the set $z$ of leaf nodes of $y$.

The next lemma gives a representation for $\operatorname{clp}(x)$ when $x$ is a finite strongly diagonal set.

Lemma 2.13. Suppose $x \subseteq{ }^{\kappa>2}$ is a finite strongly diagonal set whose meet closure is enumerated in increasing order of length as $x^{\wedge}=\left\{a_{i}: i<m\right\}$ and let $\rho$ be an increasing enumeration of $\left\{\lg \left(a_{i}\right): i<m\right\}$. Then $\operatorname{clp}(x)$ is the downwards closure of $\left\{a_{i} \circ(\rho\lceil i): i<m\}\right.$ and the mapping $a_{i} \mapsto a_{i} \circ(\rho\lceil i)$ is a strong embedding of $x^{\wedge}$ onto the meet closure of the set of leaves of $\operatorname{clp}(x)$.

Proof. Let $y$ be the downwards closure of $\left\{a_{i} \circ(\rho\lceil i): i<m\}\right.$. Then $y$ is a subtree of ${ }^{\kappa>} 2$ and the root of ${ }^{\kappa>} 2$, namely $\emptyset$, is in $y$. Since $a_{i} \circ(\rho\lceil i)$ has length $i$, it follows that $y$ has height $m$. The set of leaves of $y$ is

$$
z=\left\{a_{i} \circ\left(\rho\lceil i): i<m \wedge a_{i} \in A\right\}\right.
$$

and $z^{\wedge}=\left\{a_{i} \circ(\rho\lceil i): i<m\}\right.$. One can show that the mapping $a_{i} \mapsto a_{i} \circ(\rho\lceil i)$ is a strong embedding of $x^{\wedge}$ onto $z^{\wedge}$. Since $\operatorname{clp}(x)$ has height at least $m$, it follows that $y=\operatorname{clp}(x)$.

Lemma 2.14. Suppose $x$ and $y$ are finite strongly diagonal sets and $\Phi$ is a strong embedding of $x^{\wedge}$ onto $y^{\wedge}$. Then $\operatorname{clp}(x)=\operatorname{clp}(y)$.

Proof. Since the elements of $x^{\wedge}$ all have different lengths, and strong embeddings preserve length order, $\Phi$ is one-to-one. Enumerate $x^{\wedge}$ and $y^{\wedge}$ in increasing order of length as $x^{\wedge}=\left\{a_{i}: i<m\right\}$ and $y^{\wedge}=\left\{b_{i}: i<m\right\}$. Then $\Phi\left(a_{i}\right)=b_{i}$ for all $i<m$. Let $\rho: \mu \rightarrow\left\{\lg \left(a_{i}\right): i<m\right\}$ and $\tau: \mu \rightarrow\left\{\lg \left(b_{i}\right): i<m\right\}$ be increasing enumerations.

Use induction on $i<m$ to show that $a_{i} \circ\left(\rho\lceil i)=b_{i} \circ(\tau \mid i)\right.$. Start by noting that $a_{0} \circ\left(\rho\lceil 0)=\emptyset=b_{0} \circ\left(\tau\lceil 0)\right.\right.$. Suppose for $j<i, a_{j} \circ\left(\rho\lceil j)=b_{j} \circ(\tau\lceil j)\right.$. Then for all $j<i,\left(a_{i} \circ(\rho\lceil i))(j)=a_{i}\left(\lg \left(a_{j}\right)\right)=\Phi\left(a_{i}\right)\left(\lg \left(\Phi\left(a_{j}\right)\right)\right)=b_{i}\left(\lg \left(b_{j}\right)\right)\right.$, since $\Phi$ is a strong embedding. That is, $a_{i} \circ\left(\rho\lceil i)=b_{i} \circ(\tau\lceil i)\right.$.

Now the lemma follows by Lemma 2.13 .

The next definition identifies sufficient conditions for a map to carry a strongly diagonal set to one of the same $m$-type. The lexicographic order for us will be the partial order $<_{\operatorname{lex}}$ on ${ }^{\kappa>}{ }_{2}$ defined by $s<_{\operatorname{lex}} t$ if $s$ and $t$ are incomparable and $(s \wedge t) \frown\langle 0\rangle \subseteq s$ and $(s \wedge t) \frown\langle 1\rangle \subseteq t$. 
Definition 2.15. Call a map $f:{ }^{\kappa>} 2 \rightarrow^{\kappa>} 2$ polite if it satisfies the following conditions for all $x, y, u, v$ :

1. (preservation of lexicographic order) if $x$ and $y$ are incomparable and $x<_{\operatorname{lex}} y$, then $f(x)$ and $f(y)$ are incomparable and $f(x)<_{\operatorname{lex}} f(y)$;

2. (meet regularity) if $\{x, u, v\}$ is diagonal and $x \wedge u=x \wedge v$, then $f(x) \wedge$ $f(u)=f(x) \wedge f(v)$;

3. (preservation of meet length order) if $\lg (x \wedge y)<\lg (u \wedge v)$, then $\lg (f(x) \wedge f(y))<\lg (f(u) \wedge f(v))$.

Call it polite to strongly diagonal sets if it is a pnp map which satisfies the above conditions for all $x, y, u, v$ with $\{x, y, u, v\}$ a strongly diagonal set.

The next lemma follows immediately from the above definition.

Lemma 2.16. Strong embeddings are polite. The collection of polite embeddings is closed under composition as is the collection of embeddings polite to strongly diagonal sets.

Lemma 2.17. Suppose $\phi:{ }^{\kappa>} 2 \rightarrow{ }^{\kappa>} 2$ is a map which is polite to strongly diagonal sets and whose image is a strongly diagonal set. For any finite strongly diagonal set $A, \operatorname{clp}(A)=\operatorname{clp}(\phi[A])$ and there is a strong embedding $\bar{\phi}: A^{\wedge} \rightarrow$ $(\phi[A])^{\wedge}$ such that for all $x, y$ in $A, \bar{\phi}(x \wedge y)=\phi(x) \wedge \phi(y)$.

Proof. Fix a finite strongly diagonal set $A$. Let $\bar{\phi}(a)=\phi(a)$ for $a \in A$ and let $\bar{\phi}(a \wedge b)=\phi(a) \wedge \phi(b)$ for $a, b \in A$.

Claim 2.17.a. The map $\bar{\phi}$ is well-defined.

Proof. Suppose $x \wedge y=u \wedge v$. If $x=y$ or $u=v$, then $x=y=u=v$ since $A$ is strongly diagonal. Thus if $x=y$ or $u=v$ or $\{x, y\}=\{u, v\}$, then $\phi(x) \wedge \phi(y)=\phi(u) \wedge \phi(v)$. Otherwise, if $|\{x, y, u, v\}|=3$, then the claim follows from meet regularity. The final case is $|\{x, y, u, v\}|=4$. Since $\{x, y, u\}$ is a three element diagonal set and $x \wedge y$ is an initial segment of all three elements, either $x \wedge y=x \wedge u$ or $x \wedge y=y \wedge u$. Hence by meet regularity, either $\phi(x) \wedge \phi(y)=\phi(x) \wedge \phi(u)=\phi(u) \wedge \phi(v)$ or $\phi(x) \wedge \phi(y)=\phi(y) \wedge \phi(u)=\phi(u) \wedge \phi(v)$, and the claim follows.

Claim 2.17.b. The map $\bar{\phi}$ preserves length order.

Proof. Assume $s, t \in A^{\wedge}$ satisfy $\lg (s)<\lg (t)$. Let $x, y, u, v \in A$ be such that $s=x \wedge y$ and $t=u \wedge v$. By preservation of meet length order, $\lg (\bar{\phi}(s))=$ $\lg (\phi(x) \wedge \phi(y))<\lg (\phi(u) \wedge \phi(v))=\lg (\bar{\phi}(t))$.

Claim 2.17.c. For all $s, t$ in $A, \bar{\phi}(s \wedge t)=\phi(s) \wedge \phi(t)$ and $\bar{\phi}$ is a pnp map. 
Proof. By the definition of $\bar{\phi}$ and the previous claims, it suffices to show $\bar{\phi}$ preserves passing numbers. Suppose $s$ and $t$ are in $A^{\wedge}$ and $\lg (s)<\lg (t)$. Since any $t^{\prime} \in\left({ }^{\wedge} A\right)$ is either in $A$ or has an extension $t$ in $A$ with $\bar{\phi}\left(t^{\prime}\right) \subseteq \bar{\phi}(t)=\phi(t)$, we may assume without loss of generality that $t$ is in $A$. If $s \in A$, then the conclusion follows since $\phi$ is a pnp map. So suppose $s=x \wedge y$ for $x$ and $y$ distinct elements of $A$. Consider $s \wedge t$.

If $\lg (s \wedge t)<\lg (s)$, then $\lg (\bar{\phi}(s \wedge t))<\lg (\bar{\phi}(s))$ by the previous claim. In this case, $\bar{\phi}(t)(\lg (\bar{\phi}(s)))=0=t(\lg (s))$, since $A$ and its image under $\phi$ are both strongly diagonal.

If $\lg (s \wedge t)=\lg (s)$, then $s \subseteq t$. Let $w \in A$ be such that $s=t \wedge w$. Then the value of $t(\lg (s))$ and $\bar{\phi}(t)(\lg (\bar{\phi}(s)))$ are determined by the lexicographic order of the pairs $t, w$ and $\phi(t), \phi(w)$. Since $\phi$ preserves lexicographic order, $\bar{\phi}(t)(\lg (\bar{\phi}(s)))=t(\lg (s))$, as required.

Claim 2.17.d. The map $\bar{\phi}$ preserves extension.

Proof. Suppose $s, t \in A^{\wedge}$ and $s \subsetneq t$. Since $A$ is strongly diagonal, it follows that $s=x \wedge y$ for $x, y \in A$ with $x<_{\text {lex }} y$. Let $u, v \in A$ be such that $t=u \wedge v$, where we allow the possibility that $u=v$.

First suppose $t(\lg (s))=0$. Then $s \frown\langle 0\rangle \subseteq t$ and $x \wedge y=t \wedge y=u \wedge y=v \wedge y$. By meet regularity of $\phi, \bar{\phi}(s)=\phi(x) \wedge \phi(y)=\phi(u) \wedge \phi(y)=\phi(v) \wedge \phi(v)$. It follows that $\bar{\phi}(s) \subseteq \phi(u)$ and $\bar{\phi}(s) \subseteq \phi(v)$, so $\bar{\phi}(s) \subseteq \phi(u) \wedge \phi(v)=\bar{\phi}(t)$.

Otherwise $t(\lg (s))=1$. In this case $s \frown\langle 1\rangle \subseteq t$ and $x \wedge y=x \wedge t=x \wedge u=x \wedge v$. Argue as in the previous case to see that $\bar{s} \subseteq \phi(u) \wedge \phi(v)=\bar{\phi}(t)$.

By Claims 2.17.a, 2.17.b, 2.17.c and 2.17.d, $\bar{\phi}: A^{\wedge} \rightarrow \phi[A]^{\wedge}$ is a welldefined strong embedding. By Lemma 2.14, $\operatorname{clp}(A)=\operatorname{clp}(\phi[A])$. Since $A$ was an arbitrary strongly diagonal set, the lemma follows.

At this point we introduce a linear order of ${ }^{\kappa}>2$ into our picture.

Definition 2.18. Say that $\prec$ is a level ordering of $\kappa>2$ or alternatively that $\prec$ is an ordering of the levels of ${ }^{\kappa>} 2$, if $\prec$ linearly extends the length order, i.e. $\prec$ is a linear order of ${ }^{\kappa>} 2$ and $\lg (s)<\lg (t)$ implies $s \prec t$.

Two finite antichains $x$ and $y$ in ${ }^{\kappa>} 2$ are similar if $\operatorname{clp}(x)=\operatorname{clp}(y)$, and $\prec$-similar if the ordering $\prec$ induces the same ordering $\prec_{x}=\prec_{y}$ on the collapsed trees. In this case we call $\left(\operatorname{clp}(x), \prec_{x}\right)$ the ordered similarity type of $(x, \prec)$ and $(y, \prec)$.

Definition 2.19. Suppose $T$ is a subtree of ${ }^{\kappa>} 2$ and $D \subseteq{ }^{\kappa>} 2$ is transverse. Call $\prec$ a pre-S-vip order on $T \subseteq{ }^{\kappa>} 2$ if $\prec$ is a well-ordering of each level of $T$ such that for every $d \in S, d$ is the $\prec$-least element of its level, $T(\lg (d))$, and for all $u, v \in T(d) \backslash\{d\}), \prec$ satisfies the condition:

$$
\text { if } d \wedge u \subsetneq d \wedge v \neq d \text {, then } u \prec v \text { ). }
$$

If $D$ is diagonal, call $\prec$ a $D$-vip order if it is a pre- $D^{\wedge}$-vip order and for $u, v \in D^{\wedge}$ with $\lg (d)<\lg (u), \lg (v), \prec$ also satisfies the condition below:

$$
\text { if } d \wedge u=d \wedge v \neq d \text { and } u(\lg (d))<v(\lg (d)) \text {, then } u\lceil\lg (d) \prec v \uparrow \lg (d) \text {. }
$$


Lemma 2.20. If $S \subseteq{ }^{\kappa>} 2$ is transverse, then there is a pre-D-vip order of ${ }^{\kappa>} 2$.

Proof. Let $\lessdot$ be any well-ordering of the levels of ${ }^{\kappa>} 2$. Use recursion to define a pre- $S$-vip order $\prec$ by adjusting $\lessdot$ separately on each level which has an element of $S$.

Definition 2.21. An ordered similarity tree is a similarity tree $t$ with an ordering $\prec_{t}$ of its levels. Call $(\tau, \lessdot)$ a vip $m$-type if $\tau$ is an $m$-type and $\lessdot$ is an $L$-vip order on $\tau$. If $x$ is a subset of ${ }^{\kappa>} 2$ and $\prec$ is a given order of ${ }^{\kappa>} 2$, then $\prec_{x}$ is the order on $\operatorname{clp}(x)$ induced by the strong embedding from $\operatorname{clp}(x)$ to $x$ and $\prec$.

Lemma 2.22. Assume $D \subseteq \kappa>2$ is a strongly diagonal set and $\prec$ is an ordering of the levels of ${ }^{\kappa>} 2$ which is a D-vip order. Then for all $m$-element sets $x \subseteq D$, $\left(\operatorname{clp}(x), \prec_{x}\right)$ is a vip m-type.

A $\kappa$-dense order is an order $<_{\mathrm{Q}}$ of size $\kappa$ such that for all $A, B$ of size $<\kappa$ satisfying $A<_{Q} B$ there is $c$ with $A<_{Q} c<_{Q} B$. In [1] we defined such an order on ${ }^{\kappa>} 2$ by letting $s<<_{Q} t$ if and only if one of the following conditions holds: (1) $t \frown\langle 0\rangle \subseteq s ;(2) s \frown\langle 1\rangle \subseteq t$; or (3) $s$ and $t$ are incomparable and $s<_{\operatorname{lex}} t$. We proved the following in [1] about this linear order.

Lemma 2.23. If $S \subseteq{ }^{\kappa>} 2$ is cofinal above $w$ and transverse, then $\left(S \cap \operatorname{Cone}(w),<_{Q}\right)$ is $\kappa$-dense.

The following theorem of Shelah plays a crucial role in our technique. A detailed proof of it is provided in [1].

Theorem 2.24 (Shelah [6]). Suppose that $m<\omega$ and $\kappa$ is a cardinal which is measurable in the generic extension obtained by adding $\lambda$ Cohen subsets of $\kappa$, where $\lambda \rightarrow(\kappa)_{2^{\kappa}}^{2 m}$. Then for any coloring $d$ of the $m$-element antichains of ${ }^{\kappa>} 2$ into $\sigma<\kappa$ colors, and any well-ordering $\prec$ of the levels of ${ }^{\kappa>} 2$, there is a strong embedding $e:{ }^{\kappa>} 2 \rightarrow^{\kappa>} 2$ and $a$ dense set of elements $w$ such that

1. $e(s) \prec e(t)$ for all $s \prec t$ from $\operatorname{Cone}(w)$, and

2. $d(e[a])=d(e[b])$ for all $\prec$-similar $m$-element antichains a and $b$ of Cone $(w)$.

\section{$3 \quad$ A strong diagonalization}

In this section we prove results which will be used to obtain a limitation of colors result for $\kappa$-Rado graphs using Shelah's Theorem 2.24.

Definition 3.1. Call $f$ a pnp diagonalization into $S^{*} \cap \operatorname{Cone}(w)$ if $f$ is a polite injective $<_{\mathrm{Q}}$-preserving pnp map whose range is a strongly diagonal subset $D$ with $D^{\wedge} \subseteq S^{*} \cap \operatorname{Cone}(w)$. Call $f$ a pnp diagonalization if it is a pnp diagonalization into $S^{*}$.

An extra quality we desire for our diagonalization is level harmony, which will be used in the section on lower bounds. 
Definition 3.2. Suppose $f:{ }^{\kappa>} 2 \rightarrow^{\kappa>} 2$ is an injective map. Define $\hat{f}:{ }^{\kappa>} 2 \rightarrow$ ${ }^{\kappa>} 2$ by $\hat{f}(s)=f(s \frown\langle 0\rangle) \wedge f(s \frown\langle 1\rangle)$. Then $f$ has level harmony if $\hat{f}$ is an

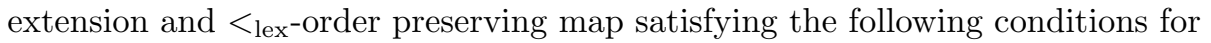
all $s, t \in{ }^{\kappa>} 2$ :

1. $\hat{f}(s) \subsetneq f(s)$;

2. $\lg (s)<\lg (t)$ implies $\lg (f(s))<\lg (\hat{f}(t))$;

3. $\lg (s)=\lg (t)$ implies $\lg (\hat{f}(s))<\lg (f(t))$.

Lemma 3.3. [Diagonalization lemma] For any $w \in{ }^{\kappa>} 2$, there is a pnp diagonalization into $S^{*} \cap$ Cone $(w)$ which has level harmony.

Proof. Our plan is to approach the problem in pieces by using recursion to define three functions, $\varphi_{0}, \varphi_{1}, \varphi:{ }^{\kappa>} 2 \rightarrow S^{*}$ so that $\varphi$ is the desired diagonalization, $\hat{\varphi}=\varphi_{0}, \varphi_{1}(t)$ is the minimal extension in $S^{*}$ of $\varphi_{0} \frown\langle 1\rangle, \varphi(t) \wedge \varphi(t \frown\langle 1\rangle)=\varphi_{1}(t)$, and $\varphi(t)<_{\operatorname{lex}} \varphi(t 八\langle 1\rangle)$.

For notational convenience, if $\varphi$ has been defined on ${ }^{\alpha>} 2$, then we let $\ell_{0}(\alpha)$ be the least $\theta$ such that $\lg (\varphi(t))<\theta$ for all $t \in{ }^{\alpha>} 2$. Also, if $\varphi_{1}$ has been defined on ${ }^{\alpha \geq 2}$, then we let $\ell_{1}(\alpha)$ be the least $\theta$ such that $\lg \left(\varphi_{1}(t)\right)<\theta$ for all $t \in{ }^{\alpha \geq 2}$.

Let $\prec$ be a well-ordering of the levels of $\kappa>2$. We use recursion on $\alpha<\kappa$ to define the restrictions to ${ }^{\alpha} 2$ of $\varphi_{0}, \varphi_{1}$ and $\varphi$ so that the following properties hold:

1. extension and lexicographic order:

(a) the restriction of $\varphi_{0}$ to ${ }^{\alpha \geq 2}$ is extension and $<_{\text {lex }}$-order preserving;

(b) for all $s \in{ }^{\alpha} 2, \varphi_{1}(s)$ is the minimal extension in $S^{*} \cap \operatorname{Cone}(w)$ of $\varphi_{0}(s) \frown\langle 1\rangle$

(c) for all $s \in{ }^{\alpha} 2, \varphi(s)$ is an extension in $S^{*} \cap \operatorname{Cone}(w)$ of $\varphi_{1}(s) \frown\langle 0\rangle$;

(d) for all $s \in{ }^{\alpha>} 2, \varphi_{0}(s \frown\langle 0\rangle)$ is an extension of $\varphi_{0}(s) \frown\langle 0\rangle$ and $\varphi_{0}(s \frown\langle 1\rangle)$ is an extension of $\varphi_{1}(s) \frown\langle 1\rangle$;

2. length order:

(a) for all $t \in{ }^{\alpha} 2$ and $s \in{ }^{\alpha \geq 2}$, if $s \prec t$, then

$\ell_{0}(\lg (s)) \leq \lg \left(\varphi_{0}(s)\right)<\lg \left(\varphi_{0}(t)\right)$ and

$\ell_{1}(\lg (s)) \leq \lg (\varphi(s))<\lg (\varphi(t))$;

3. passing number:

(a) for all $t \in{ }^{\alpha} 2$ and $s \in{ }^{\alpha \geq 2}$, if $s \prec t$ and $s \nsubseteq t$, then $\varphi_{0}(t)\left(\lg \left(\varphi_{0}(s)\right)=0\right.$ and $\varphi_{0}(t)\left(\lg \left(\varphi_{1}(s)\right)=0\right.$;

(b) for all $t \in{ }^{\alpha} 2$ and $s \in{ }^{\alpha \geq 2}$, if $s \prec t$ and $s \nsubseteq t$, then $\varphi(t)\left(\lg \left(\varphi_{0}(s)\right)=0\right.$ and $\varphi(t)\left(\lg \left(\varphi_{1}(s)\right)=0 ;\right.$

(c) for all $t \in{ }^{\alpha} 2$ and $s \in{ }^{\alpha>} 2, \varphi_{0}(t)(\lg (\varphi(s))=t(\lg (s))$;

(d) for all $s, t \in{ }^{\alpha} 2$, if $s \prec t$, then $\varphi(t)(\lg (\varphi(s))=0$. 
Suppose $\alpha<\kappa$ is arbitrary and for all $\beta<\alpha$, the restrictions to ${ }^{\beta} 2$ of $\varphi_{0}, \varphi_{1}$ and $\varphi$ have been defined. To maintain length order, we first define $\varphi_{0}$ and $\varphi_{1}$ by recursion on $\prec$ restricted to level ${ }^{\alpha} 2$. So suppose $\lg (t)=\alpha$ and for all $s \prec t$, $\varphi_{0}$ and $\varphi_{1}(s)$ have been defined.

Use extension and $<_{\text {lex }}$-order properties to identify an element $\varphi_{0}^{-}(t)$ of which $\varphi_{0}(t)$ is to be an extension by recursion. To start, for $\alpha=0$, set $\varphi_{0}^{-}(t)=\emptyset$. For $\alpha$ a limit ordinal, let $\varphi_{0}^{-}(t)$ be $\bigcup\left\{\varphi_{0}(t \mid \beta): \beta<\alpha\right\}$. For $\alpha$ a successor ordinal

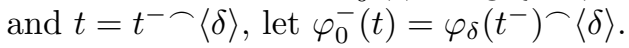

Next determine an ordinal $\gamma_{0}(t)$ sufficiently large that if $\varphi_{0}(t)$ is at least that length, it will satisfy the length order property. If $t$ is the $\prec$-least element of length $\alpha$, let $\gamma_{0}(t)=\ell_{0}(\alpha)$. If $t$ has a $\prec$-immediate predecessor $t^{\prime}$ of length $\alpha$, let $\gamma_{0}(t)=\lg \left(\varphi_{1}\left(t^{\prime}\right)\right)+1$. If $t$ is a $\prec$-limit of elements of length $\alpha$, then let $\gamma_{0}(t)$ be the supremum of $\lg \left(\varphi_{1}(s)\right)+1$ for $s$ of length $\alpha$ with $s \prec t$.

Now define an extension $\varphi_{0}^{+}(t)$ of $\varphi_{0}^{-}(t)$ of length $\gamma_{0}(t)$ so that the passing number properties are satisfied by $\varphi_{0}^{+}(t)$. If $\alpha=0$, then $t=\emptyset, \varphi_{0}^{-}(t)=\emptyset$, $\gamma_{0}(0)=0$ and $\varphi_{0}^{+}(t)=\emptyset$. If $\alpha>0$ is a limit ordinal, then by induction on $\beta<\alpha, \ell_{0}(\beta)$ is an increasing sequence. Moreover, the limit of this sequence is the length of $\varphi_{0}^{-}(t)$. It follows that $\varphi_{0}^{-}(t)$ satisfies the passing number properties for $s \in{ }^{\alpha>} 2$. Let $\varphi_{0}^{+}(t)$ be the sequence extending $\varphi_{0}^{-}(t)$ by zeros, as needed, to a length of $\gamma_{0}(t)$. If $\alpha$ is a successor ordinal and $t=t^{-} \frown\langle\delta\rangle$, then let $\varphi_{0}^{+}(t)$ be the extension of $\varphi_{0}^{-}(t)$ of length $\gamma_{0}(t)$ such that for all $\eta$ with $\lg \left(\varphi_{0}^{-}(t)\right) \leq \eta<\gamma_{0}(t)$, $\varphi_{0}^{+}(\eta)=\delta$ if $\eta=\lg (\varphi(s))$ for some $s$ with $\lg (s)+1=\alpha$, and $\varphi_{0}^{+}(\eta)=0$ otherwise.

To continue, let $\varphi_{0}(t)$ be an extension in $S^{*} \cap \operatorname{Cone}(w)$ of $\varphi^{+}(t)$ and let $\varphi_{1}(t)$ be an extension in $S^{*} \cap \operatorname{Cone}(w)$ of $\varphi_{0}(t) \frown\langle 1\rangle$ as required by the extension and lexicographic order properties. The reader may now check that the various properties hold for the restrictions of $\varphi_{0}$ and $\varphi_{1}$ to ${ }^{\alpha} 2$.

Use a similar process to define the restriction of $\varphi$ to ${ }^{\alpha} 2$ by recursion on $\prec$ restricted to ${ }^{\alpha} 2$. Suppose that $\lg (t)=\alpha$ and for all $s \prec t, \varphi(s)$ has been defined. Let $\varphi^{-}(t)=\varphi_{1}(t) \frown\langle 0\rangle$.

If $t$ is the $\prec$-least element of length $\alpha$, let $\gamma_{1}(t)=\ell_{1}(\alpha)$. If $t$ has a $\prec-$ immediate predecessor $t^{\prime}$ of length $\alpha$, let $\gamma_{1}(t)=\lg \left(\varphi\left(t^{\prime}\right)\right)+1$. If $t$ is a $\prec$-limit of elements of length $\alpha$, then let $\gamma_{1}(t)$ be the supremum of $\lg (\varphi(s))+1$ for $s$ of length $\alpha$ with $s \prec t$.

Next define an extension $\varphi^{+}(t)$ of $\varphi^{-}(t)$ of length $\gamma_{1}(t)$ so that the passing number properties are satisfied by $\varphi^{+}(t)$. If $\alpha=0$, there are no passing number properties that need be checked, and we set $\varphi^{+}(t)=\varphi^{-}(t)$. If $\alpha>0$, then let $\varphi^{+}(t)$ be the extension by zeros of $\varphi^{-}(t)$ of length $\gamma_{1}(t)$. Since $\varphi_{0}(t)$ and $\varphi_{1}(t)$ satisfy the passing numbers properties, it follows that $\varphi^{+}(t)$ does as well, since all passing numbers longer than $\lg \left(\varphi^{-}(t)\right)$ will be zero.

Finally let $\varphi(t)$ be an extension in $S^{*} \cap \operatorname{Cone}(w)$ of $\varphi^{+}(t)$. The reader may now check that the various properties hold for the restriction of $\varphi$ to ${ }^{\alpha} 2$.

This completes the recursive construction of $\varphi_{0}, \varphi_{1}$ and $\varphi$. By induction, the various properties hold for all $\alpha<\kappa$.

Thus $\varphi_{0}=\hat{\varphi}$ is extension and $<_{\text {lex }}$-order preserving, and by the length order property, different elements of the union of the ranges of $\varphi_{0}, \varphi_{1}$ and 
$\varphi$ have different lengths. It also follows that these three maps are injective. Moreover the union of their ranges is a subset of $S^{*} \cap \operatorname{Cone}(w)$. By the extension and lexicographic order properties and the length order property, $\varphi$ has level harmony.

By the passing number properties, $\varphi$ is a pnp map. By the extension and lexicographic order properties, $\varphi$ preserves $<_{\mathrm{Q}}$-order.

By the extension and lexicographic order properties, $\varphi$ carries incomparable elements into incomparable elements and preserves $<_{\text {lex }}$-order. By the length order property, $\varphi$ preserves meet length order. Since $\hat{\varphi}=\varphi_{0}$ preserves extension, $\varphi$ satisfies meet regularity. Thus $\varphi$ is polite.

From the extension and lexicographic order properties, it follows that the meet closure of $D:=\operatorname{ran}(\varphi)$ is the union of the ranges of $\varphi, \varphi_{0}$ and $\varphi_{1}$ and all elements of the range of $\varphi$ are incomparable. Hence $D$ is an antichain and $D^{\wedge} \subseteq S^{*} \cap \operatorname{Cone}(w)$ is transverse, so $D$ is diagonal. Note that passing numbers of 1 were introduced only to keep $\varphi_{0}$ extension and $<_{\text {lex }}$-order preserving, to ensure $\varphi(t)<_{\operatorname{lex}} \varphi_{1}(t)$ so that $<_{\mathrm{Q}^{-}}$-order is preserved, and to ensure $\varphi$ is a pnp map. It follows that $D^{\wedge}$ is strongly diagonal.

Therefore, $\varphi$ is the required pnp diagonalization into $S^{*} \cap \operatorname{Cone}(w)$ with level harmony.

Lemma 3.4. There are pnp maps $\left\langle\varphi_{t}: t \in{ }^{\kappa}>2\right\rangle$ with strongly diagonal ranges and a pre-S*-vip order $\prec$ such that for all $t \in{ }^{\kappa>} 2$, the following conditions hold:

1. if $x$ is a finite strongly diagonal set, then $\operatorname{clp}\left(\varphi_{t}[x]\right)=\operatorname{clp}(x)$;

2. the meet closure of the set $D_{t}:=\operatorname{ran}\left(\varphi_{t}\right)$ is a subset of $S^{*} \cap$ Cone $(t)$ disjoint from $D_{s}^{\wedge}$ for all $s \neq t$; and

3. $\prec$ is a $D_{t}$-vip order.

The maps $\varphi_{t}$ may be chosen to be pnp diagonalizations into $S^{*} \cap \operatorname{Cone}(t)$ with level harmony.

Proof. Use Lemma 2.20 to find $\prec^{*}$, a pre- $S^{*}$-vip order on ${ }^{\kappa>} 2$.

Apply the Diagonalization Lemma 3.3 to each $t \in{ }^{\kappa>} 2$ to obtain $\varphi_{t}^{*}$, a pnp diagonalization into $S^{*} \cap$ Cone $(t)$ which has level harmony. Use recursion on $\prec^{*}$ to define $\pi:{ }^{\kappa>} 2 \rightarrow{ }^{\kappa>} 2,\left\langle\varphi_{t}: t \in{ }^{\kappa>} 2\right\rangle$ and $\left\langle D_{t}: t \in{ }^{\kappa>} 2\right\rangle$ such that for all $t \in{ }^{\kappa>} 2, D_{t}:=\operatorname{ran}\left(\varphi_{t}\right), \pi(t)$ is an extension of $t$ with Cone $(\pi(t))$ disjoint from the union over all $s \prec^{*} t$ of $D_{s}^{\wedge}$. Since the order type of $\left\{s \in{ }^{\kappa>} 2: s \prec^{*} t\right\}$ is less than $\kappa$ and each $D_{s}$ is a strongly diagonal set whose meet closure is a subset of $S^{*}$, it is always possible to continue the recursion.

Use induction on the recursive construction to show that the meet closures of the sets $D_{t}$ are disjoint.

Let $D=\bigcup\left\{D_{t}^{\wedge}: t \in{ }^{\kappa>} 2\right\}$. Then $D$ is transverse since it is a subset of $S^{*}$ and $S^{*}$ is transverse. Let $\prec$ agree with $\prec^{*}$ on all pairs from different levels, and use recursion on $\alpha<\kappa$ to define $\prec$ from $\prec^{*}$ as follows. If there is no element of $D$ in ${ }^{\alpha} 2$ then $\prec$ and $\prec^{*}$ agree on ${ }^{\alpha} 2$. 
So suppose $d \in D_{t}^{\wedge}$ and $\lg (d)=\alpha$. For each $\beta<\alpha$, let $C(\beta)$ be the set of all $x \in{ }^{\alpha} 2$ such that $x\left\lceil\beta=d\left\lceil\beta\right.\right.$ and $x(\beta) \neq d(\beta)$. Since $\prec^{*}$ is a pre- $S^{*}$-vip order, if $\beta<\gamma<\alpha$, then $C(\beta) \prec^{*} C(\gamma)$ in the sense that for every element $x$ of $C(\beta)$ and $y$ of $C(\gamma)$, one has $x \prec^{*} y$. Use the fact that $D_{t}$ is a diagonal set to partition ${ }^{\alpha} 2=\{d\} \cup A_{\alpha}(0) \cup A_{\alpha}(1) \cup A_{\alpha}(2)$ into disjoint pieces where for $\delta<2, A_{\alpha}(\delta):=\left\{u \mid \alpha: u \in D_{t} \wedge u(\alpha)=\delta\right\}$. Let the restriction of $\prec$ to ${ }^{\alpha} 2$ be such that for each $\beta<\alpha$,

$$
C(\beta) \cap A_{\alpha}(0) \prec C(\beta) \cap A_{\alpha}(1) \prec C(\beta) \cap A_{\alpha}(2)
$$

and otherwise $\prec$ agrees with $\prec{ }^{*}$. Then the restriction of $\prec$ to ${ }^{\alpha} 2$ is a well-order, since the restriction of $\prec^{*}$ is and because $\prec^{*}$ is a pre- $S^{*}$-vip order.

Since the restriction of $\prec$ to each level is a well-order, it follows that $\prec$ is a well-ordering of the levels of ${ }^{\kappa>} 2$. For each $t \in{ }^{\kappa>} 2$, since $\prec^{*}$ is a pre- $S^{*}$-vip order, it follows that $\prec$ is a pre- $D_{t}^{\wedge}$-vip order, so by construction, $\prec$ is a $D_{t}$-vip order.

Since pnp diagonalizations with level harmony are polite, by Lemma 2.17, for any finite strongly diagonal set $x$, the collapse of $\varphi_{t}[x]$ is the same as the collapse of $x$.

\section{Type realization}

The computation of lower bounds for Rado graphs is more complex than the computation for $\kappa$-dense linear orders as done in [1]. We reduce the problem by showing for suitable $\kappa$ that if $D \subseteq{ }^{\kappa>} 2$ is the range of a pnp diagonalization with level harmony and $\prec$ is a $D$-vip level order, then every vip $m$-type is realized as $\left(\operatorname{clp}(x), \prec_{x}\right)$ for some $x \subseteq D$. In comparison with the corresponding theorem in [1], the proof uses a pnp diagonalization with level harmony in place of a semi-strong embedding.

We start this section with some definitions and results from [1].

Definition 4.1. Call an ordering $\prec$ of the levels of ${ }^{\kappa>} 2$ small if $\left({ }^{\alpha} 2, \prec\right)$ has order type $2^{\alpha}$ for each cardinal $\alpha<\kappa$.

Lemma 4.2. Suppose $\kappa$ is a limit cardinal, $\prec^{\prime}$ is a small ordering of the levels of ${ }^{\kappa>} 2$ and $w \in{ }^{\kappa>}$. For all $n<\omega$ and orderings $\lessdot$ of the levels of $n \geq 2$, there is an order preserving strong embedding $j$ taking $(n \geq 2, \lessdot)$ into (Cone $\left.(w), \prec^{\prime}\right)$, i.e. $s \lessdot t$ implies $j(s) \prec^{\prime} j(t)$. Furthermore, $j$ may be chosen such that for all $s$, the length of $j(s)$ is a cardinal.

If $s$ is a node of $T$ and both $s \frown\langle 0\rangle$ and $s \frown\langle 1\rangle$ have extensions in $T$, then we call $s$ a splitting node of $T$.

Definition 4.3. Call a node $w$ in a tree $T$ evenhanded in $T$ if for all $\delta<2$ it is the limit of splitting nodes $u \in T$ with $u \frown\langle\delta\rangle \subseteq w$. An almost perfect tree is a subtree $T \subseteq{ }^{\kappa>} 2$ such that the following three conditions hold:

- for every $t \in T$, there is a splitting node of $T$ above $t$; 
- every evenhanded limit of splitting nodes of $T$ is in $T$;

- for every splitting node $u \in T$ there are $\delta<2$ and a branch $b \subseteq T$ of length $\lg (b)=\kappa$ such that $b$ favors $\delta$ above $u$.

Lemma 4.4. Suppose that $S \subseteq{ }^{\kappa>} 2$ and $\left(S,<_{Q}\right)$ is $\kappa$-dense. Then

$$
T(S):=\{t \in T: S \cap \operatorname{Cone}(t) \text { is } \kappa \text {-dense }\}
$$

is an almost perfect tree.

Lemma 4.5. Suppose $S \subseteq{ }^{\kappa>} 2$ and $\left(S,<_{Q}\right)$ is $\kappa$-dense. Let $C(S)$ be the set of all limit ordinals $\alpha>0$ such that every $t \in T(S) \cap{ }^{\alpha>2}$ has proper extensions in both $S \cap^{\alpha>} 2$ and $\mathcal{W}(S) \cap^{\alpha>} 2$. Then $C(S)$ is a club of $\kappa$.

Now we are ready to state the main theorem of this section.

Theorem 4.6. Suppose that $\kappa$ is a cardinal which is measurable in the generic extension obtained by adding $\lambda$ Cohen subsets of $\kappa$, where $\lambda \rightarrow(\kappa)_{2^{\kappa}}^{6}$. Further suppose $f: \kappa>2 \rightarrow^{\kappa>} 2$ is a pnp diagonalization with level harmony, $D:=$ $f\left[{ }^{\kappa>} 2\right]$, and $\prec$ is a D-vip order of the levels of ${ }^{\kappa>} 2$. Then every vip $m$-type $(\tau, \lessdot)$ is realized as $\left(\operatorname{clp}(x), \prec_{x}\right)$ for some $x \subseteq D$.

Proof. For $t \in{ }^{\alpha} 2, i=0,1$ and $\delta=0,1$, define well-orderings $\prec_{t}^{i, \delta}$ on ${ }^{\alpha} 2$ as follows. First let $\beta_{t}^{0}=\lg (\hat{f}(t))$ and set $\beta_{t}^{1}=\lg (f(t))$. Next set $s \prec_{t}^{i, \delta} s^{\prime}$ if and only if $f\left(s^{\frown}\langle\delta\rangle\right)\left\lceil\beta_{t}^{i} \prec f\left(s^{\prime} \frown\langle\delta\rangle\right)\left\lceil\beta_{t}^{i}\right.\right.$.

Let $\prec^{\prime}$ be a fixed small well-ordering of the levels of ${ }^{\kappa>} 2$. Call a triple $\left\{s, s^{\prime}, t\right\}$ local if $\lg (s)=\lg \left(s^{\prime}\right)=\lg (t), s<_{\operatorname{lex}} s^{\prime}, t \prec^{\prime} s$, and $t \prec^{\prime} s^{\prime}$, and $s \wedge s^{\prime} \not t$.

Let $d$ be a coloring of the triples of $\kappa>2$ defined as follows: if $\left\{s, s^{\prime}, t\right\}$ is not local, let $d^{i, \delta}\left(\left\{s, s^{\prime}, t\right\}\right):=2$ and otherwise set $d^{i, \delta}\left(\left\{s, s^{\prime}, t\right\}\right):=\| s \prec^{\prime} s^{\prime} \Longleftrightarrow$ $s \prec_{t}^{i, \delta} s^{\prime} \|$. For $b=\left\{s, s^{\prime}, t\right\} \in\left[{ }^{\alpha} 2\right]^{3}$, define

$$
d(b):=\left(d^{0,0}(b), d^{0,1}(b), d^{1,0}(b), d^{1,1}(b)\right) .
$$

Apply Shelah's Theorem 2.24 to $d$ and $\prec^{\prime}$ to obtain a strong embedding $e:{ }^{\kappa>2} \rightarrow^{\kappa>} 2$ and an element $w$ so that for triples from $T:=e[\operatorname{Cone}(w)]$, the coloring depends only on the $\prec$-ordered similarity type of the triple.

Then two local triples $\left\{s, s^{\prime}, t\right\}$ and $\left\{u, u^{\prime}, v\right\}$ of $T$ are colored the same if and only if for all $i=0,1$ and $\delta=0,1$,

$$
s \prec_{t}^{i, \delta} s^{\prime} \Longleftrightarrow u \prec_{v}^{i, \delta} u^{\prime}
$$

Thus for $t \in T$, the orderings $\prec_{t}^{i, \delta}$ must always agree with one of $\prec^{\prime}$ and its converse on $T$ on pairs $\left\{s, s^{\prime}\right\} \subseteq T$ with $\left\{s, s^{\prime}, t\right\}$ local and $s \prec^{\prime} s^{\prime}$. Similarly, they must always agree with one of $\prec^{\prime}$ and its converse on $T$ on pairs $\left\{s, s^{\prime}\right\} \subseteq T$ with $\left\{s, s^{\prime}, t\right\}$ local and $s^{\prime} \prec^{\prime} s$. Since $\prec$ is a well-order, all of the orderings $\prec_{t}^{i, \delta}$ are also well-orders. Thus they always agree with $\prec^{\prime}$. 
Let $(\tau, \lessdot)$ be an arbitrary vip $m$-type. Let $L$ be the set of leaves of $\tau$. Then $\tau$ is a subtree of ${ }^{2 m-2} \geq 2$ and every level of ${ }^{2 m-2} \geq 2$ has exactly one element of $L^{\wedge}$. Extend $\lessdot$ defined on $\tau$ to ${ }^{*}$ defined on all of ${ }^{2 n \geq 2}$ in such a way that the extension is still a $L^{\wedge}$-vip order.

Apply Lemma 4.2 to get an order preserving strong embedding $j$ of $\left(2 m-2 \geq 2, \lessdot^{*}\right)$ into $\left(\right.$ Cone $\left.(w), \prec^{\prime}\right)$.

Let $\left\langle t_{\ell}: \ell \leq 2 m-2\right\rangle$ enumerate the elements of $L^{\wedge}$ in increasing order of length. Note that $\lg \left(t_{\ell}\right)=\ell$. For $\ell \leq 2 m-2$, define

$$
\beta_{\ell}:= \begin{cases}\lg \left(\hat{f}\left(e\left(j\left(t_{\ell}\right)\right)\right)\right) & \text { if } t_{\ell} \notin L, \\ \lg \left(f\left(e\left(j\left(t_{\ell}\right)\right)\right)\right) & \text { if } t_{\ell} \in L .\end{cases}
$$

Finally define $\rho: \tau \rightarrow{ }^{\kappa>} 2$ by recursion on $\ell \leq 2 m-2$. For $\ell=0$, let $\rho(\emptyset)=\hat{f}(e(j(\emptyset)))$. For $\ell>0$, consider three cases for elements of $\tau \cap{ }^{\ell} 2$. If $t_{\ell} \in L$, let $\rho\left(t_{\ell}\right)=f\left(e\left(j\left(t_{\ell}\right)\right)\right)$. If $t_{\ell} \notin L$, let $\rho\left(t_{\ell}\right)=\hat{f}\left(e\left(j\left(t_{\ell}\right)\right)\right)$. Note that in both these cases, $\beta_{\ell}=\lg \left(\rho\left(t_{\ell}\right)\right)$. If $s \in \tau \backslash L^{\wedge}$ has length $\ell$, then there is a unique immediate successor in $\tau, s \frown\langle\delta\rangle$. In this case, let $\rho(s)=f(e(j(s)) \frown\langle\delta\rangle)\left\lceil\beta_{\ell}\right.$.

Since $j$ sends $\lessdot^{*}$-increasing pairs to $\prec^{\prime}$-increasing pairs and $e$ is a $\prec^{\prime}$-order preserving strong embedding, their composition sends sends $\gtrless^{*}$-increasing pairs to $\prec^{\prime}$-increasing pairs. Since for $v_{\ell}=e\left(j\left(t_{\ell}\right)\right) \in T$, the order $\prec^{\prime}$ agrees with $\prec_{v_{\ell}}^{i}$ on $T \cap{ }^{\gamma} 2$ where $\gamma=\lg \left(v_{\ell}\right)$, it follows that $\rho$ sends $\lessdot^{*}$-increasing pairs to $\prec$-increasing pairs.

Since $\hat{f}$ preserves extension and lexicographic order and $\hat{f}(s) \subsetneq f(s), \rho$ preserves extension and lexicographic order. By construction $\rho$ sends levels to levels, meets to meets and leaves to leaves. Let $x=\rho[L]$ be the image under $\rho$ of the leaves of $\tau$. By construction, $x \subseteq \operatorname{ran}(f)$. Also $\left(\operatorname{clp}(x),<_{x}\right)=(\tau, \lessdot)$, as required.

Since $(\tau, \lessdot)$ was arbitrary, the theorem follows.

\section{Lower bounds for Rado graphs}

The main result of this section is Theorem 5.6 below and its Corollary 5.7, which will be used to prove the persistency property of the canonical partition described in the proof of the main theorem. Throughout this section we fix pnp diagonalizations $\left\langle\varphi_{t}: t \in{ }^{\kappa>} 2\right\rangle$ with level harmony into $S^{*}$ and a pre- $S^{*}$-vip order $\prec$ satisfying the three conditions of Lemma 3.4. For notational convenience, let $\psi=\varphi_{\emptyset}$.

To prove Theorem 5.6 we work by successive approximation.

Lemma 5.1. Suppose $f$ is a pnp diagonalization with level harmony, $g$ has range a strongly diagonal set $D$ with $D^{\wedge} \subseteq S^{*}$, and $g$ is polite to subsets of the range of $f$. Then $g \circ f$ is a pnp diagonalization with level harmony.

Proof. Since $f$ is a pnp diagonalization, it is polite, so its composition with $g$ is a polite pnp map whose range is strongly diagonal subset. Since $<_{Q}$ agrees with the lexicographic order on incomparable pairs, $f$ preserves $<_{\mathrm{Q}}$ and sends 
all pairs to incomparable pairs, the composition $g \circ f$ preserves $<_{\mathrm{Q}}$. Since $f$ is injective with range a strongly diagonal set and $g$ is a pnp map, the composition is injective. Thus the composition, $g \circ f$, is a pnp diagonalization.

For notational convenience, let $h=g \circ f$. Then

$$
\hat{h}(s):=h(s \frown\langle 0\rangle) \wedge h(s \frown\langle 1\rangle)=f(g(s \frown\langle 0\rangle)) \wedge f(g(s \frown\langle 1\rangle)) .
$$

Claim 5.1.a. For any $s \in{ }^{\kappa} 2, \hat{h}(s)=h(s \frown\langle 1-\delta\rangle) \wedge h(s) \subsetneq h(s)$, where $\delta=g(s)(\hat{g}(s))$.

Proof. Since $f$ has level harmony, $\hat{f}(s) \subsetneq f(s)$. Set $\delta:=f(s)(\lg (\hat{f}(s))$. Since $s \frown\langle 0\rangle<_{\text {lex }} s \frown\langle 1\rangle$ and $g$ preserves lexicographic order, it follows that $f(s \frown\langle 0\rangle)<_{\operatorname{lex}} f(s \frown\langle 1\rangle)$, hence $f(s \frown\langle\delta\rangle)(\lg (\hat{f}(s))=\delta$.

Since $g$ is polite, $g(f(s \frown\langle 1-\delta\rangle)) \wedge g(f(s \frown\langle\delta\rangle))=g(f(s \frown\langle 1-\delta\rangle)) \wedge g(f(s))$, by meet regularity. That is, $\hat{h}(s)=h(s \frown\langle 1-\delta\rangle) \wedge h(s)$.

Claim 5.1.b. The function $\hat{h}$ preserves lexicographic order.

Proof. Suppose $s<_{\text {lex }} t$. Since $f$ has level harmony, $\hat{f}(s)<_{\operatorname{lex}} \hat{f}(t), \hat{f}(s) \subsetneq f(s)$ and $\hat{f}(t) \subsetneq f(t)$. Hence $\hat{f}(s) \wedge \hat{f}(t)=f(s) \wedge f(t)$. Thus $\lg (f(s) \wedge f(t))<$ $\lg (f(s \frown\langle 0\rangle) \wedge f(s \frown\langle 0\rangle))$ and $\lg (f(s) \wedge f(t))<\lg (f(t \frown\langle 0\rangle) \wedge f(t \frown\langle 0\rangle))$. Since $g$ preserves meet length order, it follows that $\lg (h(s) \wedge h(t))<\lg (\hat{h}(s))$ and $\lg (h(s) \wedge h(t))<\lg (\hat{h}(t))$.

Since $g$ preserves lexicographic order, $h(s)<_{\text {lex }} h(t)$. By the Claim 5.1.a, $\hat{h}(s) \subsetneq h(s)$ and $\hat{h}(t) \subsetneq h(t)$. Thus $\hat{h}(s)<_{\operatorname{lex}} \hat{h}(t)$.

Claim 5.1.c. For all $s$ and $t, \lg (s)<\lg (t)$ implies $\lg (h(s))<\lg (\hat{h}(t))$.

Proof. Suppose $\lg (s)<\lg (t)$. Then $\lg (f(s))<\lg (\hat{f}(t))=\lg (f(s \frown\langle 0\rangle) \wedge f(s \frown\langle 1\rangle)$. Since $f(s) \wedge f(s)=f(s)$, by preservation of meet length order by $g$, it follows that $\lg (h(s)<\lg (\hat{h}(t))$.

Claim 5.1.d. For all $s$ and $t, \lg (s)=\lg (t)$ implies $\lg (\hat{h}(s))<\lg (h(t))$.

Proof. Suppose $\lg (s)=\lg (t)$. Then $\lg (\hat{f}(s))<\lg (f(t))$. Argue as in the previous claim: by preservation of meet length order by $g$, it follows that $\lg (\hat{h}(s)<$ $\lg (h(t))$.

Claim 5.1.e. The function $\hat{h}$ preserves extension.

Proof. Suppose $s \subsetneq t$. Then $\hat{f}(s) \subsetneq \hat{f}(t)$, since $f$ has level harmony. Since $g$ satisfies preservation of meet length order, $\lg (\hat{h}(s))<\lg (\hat{f}(s))$. By Claim 5.1.a, $\hat{h}(t) \subsetneq h(t)$, so $\lg (\hat{h}(s))<\lg (h(t))$. Thus to show $\hat{h}(s) \subseteq \hat{h}(t)$, it is enough to show $\hat{h}(s) \subseteq h(t)$.

For the first case, assume $h(t)(\lg (\hat{h}(s)))=1$, then $\hat{h}(s) \subseteq h(t)$, since $\operatorname{ran}(h)$ is strongly diagonal. If $t$ is one of $s \frown\langle 0\rangle$ and $s \frown\langle 1\rangle$, then $\hat{h}(s) \subseteq h(t)$ by definition of $\hat{h}(s)$. 
Next suppose $h(t)(\lg (\hat{h}(s)))=0$ and $\lg (t)>\lg (s)+1$. Since $f$ has level harmony, $\hat{f}(s) \subsetneq f(s)$ and $\hat{f}(s) \subseteq f \hat{(t)} \subsetneq f(t)$. By Claim 5.1.a, $\hat{h}(s)=h(t \frown\langle 1-$ $\delta\rangle) \wedge h(s)$ where $\delta=f(\lg (\hat{f}(s))$. For notational convenience, let $\alpha=\lg (\hat{f}(s))$. For the first subcase, suppose $f(t)(\alpha) \neq f(s)(\alpha)$. Then $f(t)(\alpha)=f(s \frown\langle 1-$ $\delta\rangle)(\alpha)$, so $f(s) \wedge f(s \frown\langle 1-\delta\rangle)=f(s) \wedge f(t)$. Since $\operatorname{ran}(f)$ is strongly diagonal and $g$ is polite, it follows that $g(f(s)) \wedge g(f(s \frown\langle 1-\delta\rangle)=g(f(s)) \wedge g(f(t))$, so $\hat{h}(s)=h(s) \wedge h(t) \subseteq h(t)$. For the second subcase, in which $f(t)(\alpha)=f(s)(\alpha)$, interchange the roles of $f(s)$ and $f(s \frown\langle 1-\delta\rangle)$. The parallel argument concludes with the inclusion $\hat{h}(s)=h(s \frown\langle 1-\delta\rangle) \wedge h(t) \subseteq h(t)$.

Now the lemma follows from the claims.

The next lemma gives an inequality, for pnp maps, which compares lengths of meets of images with lengths of images of meets.

Lemma 5.2. Suppose $g:{ }^{\kappa>} 2 \rightarrow{ }^{\kappa>} 2$ is a pnp map and $\{x, u, v\}$ is a three element strongly diagonal set with $x \wedge u=x \wedge v \subsetneq u \wedge v$. Then $\lg (g(x) \wedge g(u)) \leq$ $\lg (g(x \wedge u))$ and $\lg (g(x) \wedge g(v)) \leq \lg (g(x \wedge u))$.

Proof. Let $\alpha:=\lg (u \wedge x)=\lg (u \wedge v)$ and set $\beta:=\lg (g(x \wedge u))=\lg (g(x \wedge v)$. Since $g$ is a pnp map, $\beta<\lg (g(x))$ and $g(x)(\beta)=x(\alpha)$. Similarly, $\beta<\lg (g(u))$ and $g(u)(\beta)=u(\alpha)$. Also, $\beta<\lg (g(v))$ and $g(v)(\beta)=v(\alpha)$.

Since $x \wedge u=x \wedge v$, it follows that $x(\alpha) \neq u(\alpha)=v(\alpha)$. Consequently, $g(x)(\beta) \neq g(u)(\beta)=g(v)(\beta)$. Thus $\lg (g(x) \wedge g(u)) \leq \beta$ and $\lg (g(x) \wedge g(v)) \leq \beta$, so the lemma follows.

Call $x \in\left[{ }^{\kappa>} 2\right]^{3}$ a short leaf triple if it is strongly diagonal and some leaf of $x$ is shorter than the meet of a pair of leaves from $x$; it is a long leaf triple if it is strongly diagonal but not a short leaf subset.

Lemma 5.3. Suppose $g$ is a pnp map which preserves $<_{Q}$ and has range a strongly diagonal set. If $x$ is a long leaf triple, then $g[x]$ is a long leaf triple

Proof. List $x$ in increasing lexicographic order as $x_{0}, x_{1}, x_{2}$ and in increasing order of length as $x_{i}, x_{k}, x_{\ell}$. Then $x_{0}, x_{0} \wedge x_{1}, x_{1}, x_{1} \wedge x_{2}, x_{2}$ lists the elements of $x^{\wedge}$ in $<_{Q}$-increasing order, so $x_{0} \wedge x_{1} \neq x_{1} \wedge x_{2}$ and $x_{0} \wedge x_{2}$ is the shorter of $x_{0} \wedge x_{1}$ and $x_{1} \wedge x_{2}$. It follows that for some $\delta \in\{0,2\}$, the following is a list of the elements of $x^{\wedge}$ in increasing length order:

$$
x_{0} \wedge x_{2}, x_{1} \wedge x_{\delta}, x_{i}, x_{k}, x_{\ell} .
$$

It follows that $\lg \left(x_{0} \wedge x_{1}\right)<\lg \left(x_{2}\right)$ and $\lg \left(x_{1} \wedge x_{2}\right)<\lg \left(x_{0}\right)$. By Lemma 5.2 and the pnp property of $g, \lg \left(g\left(x_{0}\right) \wedge g\left(x_{1}\right)\right) \leq \lg \left(g\left(x_{0} \wedge x_{1}\right)\right)<\lg \left(g\left(x_{2}\right)\right)$ and $\lg \left(g\left(x_{1}\right) \wedge g\left(x_{2}\right)\right) \leq \lg \left(g\left(x_{1} \wedge x_{2}\right)\right)<\lg \left(g\left(x_{0}\right)\right)$, so $\left\{g\left(x_{0}\right), g\left(x_{1}\right), g\left(x_{2}\right)\right\}$ is a long leaf triple.

Lemma 5.4. Suppose $g$ is a pnp map which preserves $<_{Q}$ and has range a strongly diagonal set and $x$ is a long leaf triple which witnesses meet regularity. Then $\operatorname{clp}(g[x])=\operatorname{clp}(x)$. 
Proof. List $x$ as $x_{0}, x_{1}, x_{2}$ in increasing lexicographic order length order and as $x_{i}, x_{k}, x_{\ell}$ in increasing order of length. By Lemma $5.3, g[x]$ is a long leaf triple. Thus if $x_{0} \wedge x_{2}=x_{1} \wedge x_{2-\delta}, x_{1} \wedge x_{\delta}, x_{i}, x_{j}, x_{k}$ lists the meet closure of $x$ in increasing length order for some $\delta \in\{0,2\}$, then $g\left(x_{0}\right) \wedge g\left(x_{2}\right), g\left(x_{1}\right) \wedge g\left(x_{\delta}\right)$, $g\left(x_{i}\right), g\left(x_{j}\right), g\left(x_{k}\right)$ lists the meet closure of $g[x]$ in increasing length order. Thus $g$ preserves lexicographic order and meet length order on $x$ and satisfies meet regularity there, so by Lemma $2.17, \operatorname{clp}(g[x])=\operatorname{clp}(x)$.

Next we show how to use Shelah's Theorem 2.24 to obtain a polite pnp map from a $<_{Q}$-preserving pnp map whose range is a strongly diagonal set.

Lemma 5.5. Suppose that $\kappa$ is a cardinal which is measurable in the generic extension obtained by adding $\lambda$ Cohen subsets of $\kappa$, where $\lambda \rightarrow(\kappa)_{2^{\kappa}}^{6}$. Further suppose $g:{ }^{\kappa>} 2 \rightarrow{ }^{\kappa>} 2$ is a pnp map whose range is a strongly diagonal set. Then there is a pnp map $f$ such that $g \circ f$ is a polite pnp map.

Proof. Enumerate the vip 3-types as $\left(\tau_{0}, \lessdot_{0}\right), \ldots,\left(\tau_{r-1}, \lessdot_{r-1}\right)$. Define a coloring $d^{\prime}$ on three element subsets of ${ }^{\kappa} 2$ as follows:

$$
d^{\prime}(x)=\left(\left(\operatorname{clp}(x), \prec_{x}\right),\left(\operatorname{clp}(g[x]), \prec_{g[x]}\right)\right) .
$$

Apply Shelah's Theorem 2.24 to $d^{\prime}$ and $\prec$ to obtain a strong embedding $e$ and $w \in{ }^{\kappa} 2$ such that $e$ preserves $\prec$ on Cone $(w)$ and for all three element subsets $x$ of Cone $(w)$, the value of $d^{\prime}(e[x])$ depends only on the $\prec$-ordered similarity type of $x$, which is the same as the $\prec$-ordered similarity type of $e[x]$. Let $e^{\prime}=e \circ \varphi_{w}$ and set $T=\operatorname{ran}\left(e^{\prime}\right)$. Observe that for every triple $x \subseteq T\left(\operatorname{clp}(x), \prec_{x}\right)$ is a vip 3-type, since the map $\varphi_{w}$ is pnp diagonalization with level harmony into $S^{*} \cap \operatorname{Cone}(w)$ and $e$ preserves ordered similarity types. Since $\prec$ is a $D$-vip level order, for every triple $x \subseteq T,\left(\operatorname{clp}(g[x]), \prec_{g[x]}\right)$ is a vip 3-type. Define $d^{\prime \prime}$ on $T$ by by $d^{\prime \prime}(x)=d^{\prime}\left(\varphi_{w}[a]\right)$ where $x=e\left(\varphi_{w}(a)\right)$. Since the value of $d^{\prime}$ on $y \subseteq e[\operatorname{Cone}(w)]$ depends only on $\left(\operatorname{clp}(y), \prec_{y}\right)$, the value of $d^{\prime \prime}$ on $x \in[T]^{3}$ depends only on $\left(\operatorname{clp}(x), \prec_{x}\right)$.

To show $g$ satisfies meet regularity on $T$ it is enough to show it satisfies it for every triple from $g$. Next we single out the key property. Suppose $x_{0}, x_{1}, x_{2}$ lists a strongly diagonal set $x$ in increasing lexicographic order. Then $x$ witnesses meet regularity for $g$ if either $\left(x_{0} \wedge x_{2}=x_{0} \wedge x_{1}\right.$ and $\left.g\left(x_{0}\right) \wedge g\left(x_{2}\right)=g\left(x_{0}\right) \wedge g\left(x_{1}\right)\right)$ or $\left(x_{0} \wedge x_{2}=x_{1} \wedge x_{2}\right.$ and $\left.g\left(x_{0}\right) \wedge g\left(x_{2}\right)=g\left(x_{1}\right) \wedge g\left(x_{2}\right)\right)$. It refutes meet regularity for $g$ if either $\left(x_{0} \wedge x_{2}=x_{0} \wedge x_{1}\right.$ and $\left.g\left(x_{0}\right) \wedge g\left(x_{2}\right) \neq g\left(x_{0}\right) \wedge g\left(x_{1}\right)\right)$ or $\left(x_{0} \wedge x_{2}=x_{1} \wedge x_{2}\right.$ and $\left.g\left(x_{0}\right) \wedge g\left(x_{2}\right) \neq g\left(x_{1}\right) \wedge g\left(x_{2}\right)\right)$.

Claim 5.5.a. Every short leaf triple $x \subseteq T$ witnesses meet regularity; also its image $g[x]$ is a short leaf triple and $g$ preserves meet length order on $x$.

Proof. Let $(\tau, \lessdot)$ a vip 3-type with $\tau$ a short leaf triple. List the leaves of $\tau$ in increasing order of length as $a_{0}, a_{1}, a_{2}$. Since $\tau$ is a short leaf triple, the meet closure of the set of leaves of $\tau$ may be listed in increasing length order as follows:

$$
a_{0} \wedge a_{1}=a_{0} \wedge a_{1}, a_{0}, a_{1} \wedge a_{2}, a_{1}, a_{2} .
$$


Thus $a_{0} \wedge a_{1}=\emptyset=a_{0} \wedge a_{2}, \lg \left(a_{0}\right)=1, \lg \left(a_{0} \wedge a_{1}\right)=2, \lg \left(a_{1}\right)=3$ and $\lg \left(a_{2}\right)=4$.

We claim that every $x \subseteq T$ with $\operatorname{clp}(x)=\tau$ witnesses meet regularity. We start by building a large family of triples whose image under $e^{\prime}$ has ordered similarity type $(\tau, \lessdot)$. Let $\nu$ be a cardinal larger than $\lg \left(g\left(e^{\prime}\left(a_{0}\right)\right)\right)$. For $\alpha<\nu$, let $b_{\alpha}$ be the sequence of length $2 \alpha+3$ if $\alpha<\omega$ and of length $\gamma+2 n+1$ if $\alpha=\gamma+n$ for some limit ordinal $\gamma \geq \omega$ and $n<\omega$ such that

$$
b_{\alpha}(\eta)= \begin{cases}a_{1}(0), & \text { if } \eta=0 \\ a_{1}(1), & \text { if } \eta=1 \\ a_{2}(2), & \text { if } 0<\eta<\lg \left(b_{\alpha}\right)-1 \text { even } \\ a_{1}(2), & \text { if } \eta=\lg \left(b_{\alpha}\right)-1 \\ a_{2}(3), & \text { otherwise }\end{cases}
$$

Note that the length of $b_{\alpha}$ is always odd and at least 3, and that for odd $\eta \geq 3, b_{\alpha}(\eta)=a_{2}(3)$. For $\beta<\alpha$, by construction, $b_{\beta} \wedge b_{\alpha}=b_{\beta}\left\lceil\left(\lg \left(b_{\beta}\right)-\right.\right.$ $1)$. Using the above calculations, the reader may check that for $\beta<\alpha$, one has $\operatorname{clp}\left(\left\{a_{0}, b_{\beta}, b_{\alpha}\right\}\right)=\tau$. Notice that $a_{0} \wedge b_{\beta}=a_{0} \wedge b_{\alpha}=\emptyset$. Since $\varphi_{w}$ is a pnp diagonalization, $\prec$ is a $\operatorname{ran}\left(\varphi_{w}\right)$-vip level order, and $\lessdot$ is the only level order on $\tau$ which makes it a vip 3-type, the ordered similarity type of $\left\{\varphi_{w}\left(a_{0}\right), \varphi_{w}\left(b_{\beta}\right), \varphi_{w}\left(b_{\alpha}\right)\right\}$ is $(\tau, \lessdot)$. Since $e$ is a strong embedding which preserves $\prec$ on Cone $(w)$, the ordered similarity type of set $x(\beta, \alpha):=\left\{e^{\prime}\left(a_{0}\right), e^{\prime}\left(b_{\beta}\right), e^{\prime}\left(b_{\alpha}\right)\right\}$ is also $(\tau, \lessdot)$.

Thus each $x(\beta, \alpha)$ either witnesses or refutes meet regularity for $g$. Since $d^{\prime \prime}$ is constant on triples from $T$ of the same ordered similarity type, and every $x(\beta, \alpha)$ has ordered similarity type $(\tau, \lessdot)$, either every $x(\beta, \alpha)$ witnesses meet regularity or every $x(\beta, \alpha)$ refutes it.

Suppose each $x(\beta, \alpha)$ refutes meet regularity. Then $g\left(e^{\prime}\left(a_{0}\right)\right) \wedge g\left(e^{\prime}\left(b_{\beta}\right)\right) \neq$ $g\left(e^{\prime}\left(a_{0}\right)\right) \wedge g\left(e^{\prime}\left(b_{\alpha}\right)\right)$ for $\beta<\alpha<\nu$. Since $\nu$ is a cardinal larger than $\lg \left(g\left(e\left(a_{0}\right)\right)\right)$, by the Pigeonhole Principle, there are $\beta<\alpha$ with $g\left(e^{\prime}\left(a_{0}\right)\right) \wedge g\left(e^{\prime}\left(b_{\beta}\right)\right)=$ $g\left(e^{\prime}\left(a_{0}\right)\right) \wedge g\left(e^{\prime}\left(b_{\alpha}\right)\right)$. This contradiction shows that each $x(\beta, \alpha)$ witnesses meet regularity. It follows that every $x \subseteq T$ with $\operatorname{clp}(x)=\tau$ witnesses meet regularity.

Next we claim that for every $x \subseteq T$ with $\operatorname{clp}(x)=\tau, g(x)$ is a short leaf triple and $g$ preserves meet length order on $x$. Assume toward a contradiction that $g(x)$ is not a short leaf triple. Let $\left(\tau^{\prime}, \lessdot^{\prime}\right)$ be the ordered similarity type of $g(x)$. Since $g(x)$ is strongly diagonal, it must be a long leaf triple. List the leaves of $x$ in increasing length order as $x_{0}, x_{1}, x_{2}$. Since $x$ witnesses meet regularity for $g$, the meet closure of $x$ may be listed in increasing length order: $g\left(x_{0}\right) \wedge$ $g\left(x_{1}\right)=g\left(x_{0}\right) \wedge g\left(x_{2}\right), g\left(x_{1}\right) \wedge g\left(x_{2}\right), g\left(x_{0}\right), g\left(x_{1}\right), g\left(x_{2}\right)$. Let $\nu$ be an uncountable cardinal greater than $2^{\left|\lg \left(g\left(e^{\prime}\left(a_{0}\right)\right)\right)\right|}$. Construct a sequence $\left\langle b_{\alpha}: \alpha<\nu\right\rangle$ as in the previous claim such that for all $\alpha<\nu, a_{0} \wedge b_{\alpha}=\emptyset$ and for all $\beta<\alpha<\nu$, $\operatorname{clp}\left(\left\{a_{0}, b_{\beta}, b_{\alpha}\right\}\right)=\tau$ and $\lg \left(b_{\beta}\right)<\lg \left(b_{\alpha}\right)$. As we saw in the earlier claim, for $\beta<\alpha<\nu$ and for $\left\{\varphi_{w}\left(a_{0}\right), \varphi_{w}\left(b_{\beta}\right), \varphi_{w}\left(b_{\alpha}\right)\right\}$ has ordered similarity type $(\tau, \lessdot)$ as does $x(\beta, \alpha):=\left\{e^{\prime}\left(a_{0}\right), e^{\prime}\left(b_{\beta}\right), e^{\prime}\left(b_{\alpha}\right)\right\}$.

Since $d^{\prime \prime}$ is constant on triples of $T$ of the same ordered similarity type, each $g[x(\beta, \alpha)]$ has ordered similarity type $\left(\tau^{\prime}, \lessdot^{\prime}\right)$. Thus for all $\beta<\alpha<\nu$, 
the inequality $\lg \left(g\left(e^{\prime}\left(a_{0}\right)\right)\right) \geq \lg \left(g\left(e^{\prime}\left(a_{\beta}\right)\right) \wedge g\left(e^{\prime}\left(a_{\alpha}\right)\right)\right)$ holds. Since the range of $g$ is a strongly diagonal set, these inequalities must be strict. Since $\nu$ is an uncountable cardinal greater than $2^{\left|\lg \left(g\left(a_{0}\right)\right)\right|}$, by the Pigeonhole Principle, there are $\beta<\gamma$ with

$$
g\left(e^{\prime}\left(a_{\beta}\right)\right)\left\lceil\lg \left(g\left(e^{\prime}\left(a_{0}\right)\right)\right)=g\left(e^{\prime}\left(a_{\gamma}\right)\right)\left\lceil\lg \left(g\left(e^{\prime}\left(a_{0}\right)\right)\right) .\right.\right.
$$

Thus we have reached the contradiction that $g\left(e^{\prime}\left(a_{\beta}\right)\right) \wedge g\left(e^{\prime}\left(a_{\gamma}\right)\right)$ must have length shorter than and greater than or equal to $\lg \left(g\left(e^{\prime}\left(a_{0}\right)\right)\right)$. Thus $g(x)$ is a short leaf triple.

Since $g(x)$ is a short leaf triple and $g$ preserves length order, the elements of the meet closure of $g(x)$ may be listed in increasing length order:

$$
g\left(x_{0}\right) \wedge g\left(x_{1}\right)=g\left(x_{0}\right) \wedge g\left(x_{2}\right), g\left(x_{0}\right), g\left(x_{1}\right) \wedge g\left(x_{2}\right), g\left(x_{1}\right), g\left(x_{2}\right) .
$$

Thus $g$ preserves meet length order on $x$.

We have shown that every short leaf triple which realizes $(\tau, \lessdot)$ witnesses meet regularity for $g$, that $g$ preserves meet length order on it, and that the image under $g$ is a short leaf triple. Since $(\tau, \lessdot)$ was an arbitrary vip 3-type of a short leaf triple, the claim now follows

Claim 5.5.b. Every triple $x \subseteq T$ witnesses meet regularity for $g$.

Proof. Let $(\tau, \lessdot)$ be an arbitrary vip 3-type. If $\tau$ is a short leaf triple, then every triple $x$ with $\operatorname{clp}(x)=\tau$ witnesses meet regularity for $g$ and $g$ preserves meet length order on $x$ by Claim 5.5.a.

So assume $\tau$ is a long leaf triple. Let the leaves of $\tau$ be listing in increasing lexicographic order as $a_{0}, a_{1}, a_{2}$. Since $\tau$ is strongly diagonal, either $a_{0} \wedge a_{2}=$ $a_{0} \wedge a_{1}$ or $a_{0} \wedge a_{2}=a_{1} \wedge a_{2}$.

For the first case, suppose $a_{0} \wedge a_{2}=a_{0} \wedge a_{1}$.

Then $a_{0} \wedge a_{1}=\emptyset$ and $a_{1} \wedge a_{2}=\langle 1\rangle$, since $\tau$ is a long leaf triple. Let $s^{*}=\langle 1,0\rangle, t^{*}=\langle 0,0,0,1\rangle, u_{0}^{*}=\langle 0,0,0,0\rangle \frown a_{0}, u_{1}^{*}=\langle 1,1,0,0\rangle \frown a_{1}$, and $u_{2}^{*}=$ $\langle 1,1,0,0,0\rangle \frown a_{2}$. Let $z^{*}=\left\{s^{*}, t^{*}, u_{0}^{*}, u_{1}^{*}, u_{2}^{*}\right\}$. Note that the lengths of the meet closure listed in increasing order are

$$
s^{*} \wedge t^{*}, s^{*} \wedge u_{1}^{*}, s^{*}, u_{0}^{*} \wedge t^{*}, t^{*}, u_{1}^{*} \wedge u_{2}^{*}, u_{i}^{*}, u_{j}^{*}, u_{k}^{*}
$$

where $u_{i}^{*}, u_{j}^{*}, u_{k}^{*}$ lists $u_{0}^{*}, u_{1}^{*}, u_{2}^{*}$ in increasing order of length. Thus $z^{*}$ is strongly diagonal and $\operatorname{clp}\left(z^{*}\right)=z^{*}$. Let $\prec^{*}$ be a vip ordering of the levels of $\operatorname{clp}\left(z^{*}\right)$ such that if $\lg \left(a_{0}\right)<\min \left\{\lg \left(a_{1}\right), \lg \left(a_{2}\right)\right\}$, then $u_{1}^{*}\left\lceil\lg \left(u_{0}^{*}\right) \prec^{*} u_{2}^{*}\left\lceil\lg \left(u_{0}^{*}\right)\right.\right.$ if and only if $a_{1}\left\lceil\lg \left(a_{0}\right) \lessdot a_{2}\left\lceil\lg \left(a_{0}\right)\right.\right.$. The careful reader may check that there is one and only one way to define $\prec^{*}$.

By Theorem 4.6, the vip 5-type $\left(z^{*}, \prec^{*}\right)$ can be realized by a subset $z=$ $\left\{u_{0}, u_{1}, u_{2}, s, t\right\}$ of the range of $\varphi_{w}$. Note for $u=\left\{u_{0}, u_{1}, u_{2}\right\},\left(\operatorname{clp}(u), \prec_{u}\right)=$ $(\tau, \lessdot)=\left(\operatorname{clp}(e[u]), \prec_{e[u]}\right)$. Moreover, $\left\{e\left(u_{0}\right), e(s), e(t)\right\}$ and $\left\{e(t), e\left(u_{1}\right), e\left(u_{2}\right)\right\}$ are short leaf triples, so by Claim 5.5.a, the inequalities $\lg (g(e(t)))<\lg \left(g\left(e\left(u_{1}\right)\right) \wedge g\left(e\left(u_{2}\right)\right)\right)$ and $\lg (g(e(s)))<\lg (g(e(t)))$ hold. Since $e\left(u_{0}\right) \wedge e\left(u_{1}\right)=e\left(u_{0}\right) \wedge e\left(u_{2}\right) \subsetneq e\left(u_{1}\right) \wedge e\left(u_{2}\right)$, by Lemma 5.2, 
$\lg \left(g\left(e\left(u_{0}\right)\right) \wedge g\left(e\left(u_{1}\right)\right)\right) \leq \lg \left(g\left(e\left(u_{0} \wedge u_{1}\right)\right)\right)$ and

$\lg \left(g\left(e\left(u_{0}\right)\right) \wedge g\left(e\left(u_{2}\right)\right)\right) \leq \lg \left(g\left(e\left(u_{0} \wedge u_{1}\right)\right)\right)$.

By construction, $u_{0} \wedge u_{1}=s \wedge t$, so $e\left(u_{0} \wedge u_{1}\right)=e(s \wedge t)$ and $g\left(e\left(u_{0} \wedge\right.\right.$ $\left.\left.u_{1}\right)\right)=g(e(s \wedge t))$ is shorter than $g(e(t))$. Therefore $\lg \left(g\left(e\left(u_{0}\right)\right) \wedge g\left(e\left(u_{1}\right)\right)\right) \leq$ $\lg (g(e(s \wedge t)))<\lg \left(g(e((t)))<\lg \left(g\left(e\left(u_{1}\right)\right) \wedge g\left(e\left(u_{2}\right)\right)\right)\right.$. Similarly, $\lg \left(g\left(e\left(u_{0}\right)\right) \wedge\right.$ $\left.g\left(e\left(u_{2}\right)\right)\right)<\lg \left(g\left(e\left(u_{1}\right)\right) \wedge g\left(e\left(u_{2}\right)\right)\right)$. Thus $e[u]$ witnesses meet regularity for $g$ and $\left(\operatorname{clp}(e[u]), \prec_{e[u]}\right)=(\tau, \lessdot)$. It follows that any $x \subseteq T$ with $\left(\operatorname{clp}(x), \prec_{x}\right)=(\tau, \lessdot)$ also witnesses meet regularity.

Our next goal is to show that for every pair from $T$, depending on the type of the pair, $g$ preserves or reverses the lexicographic order of the pair.

For notational convenience, let $\eta_{0}$ be the 2-type whose leaves are $\langle 0\rangle$ and $\langle 1,0\rangle$; let $\eta_{1}$ be the 2 -type whose leaves are $\langle 1\rangle$ and $\langle 0,0\rangle$; let $\eta_{2}$ be the 2 -type whose leaves are $\langle 0\rangle$ and $\langle 1,1\rangle$; and let $\eta_{3}$ be the 2 -type whose leaves are $\langle 1\rangle$ and $\langle 0,1\rangle$.

For all $x \in\left[{ }^{\kappa} 2\right]^{2}$, the set $\operatorname{clp}(x)$ is one of $\eta_{0}, \eta_{1}, \eta_{2}$ and $\eta_{3}$. For $i<4$, let $\triangleleft_{i}$ be the unique vip level order on $\eta_{i}$. Then the set $\left\{\left(\eta_{i}, \triangleleft_{i}\right): i<4\right\}$ is the collection of all vip 2-types.

Since $g$ is a pnp map and $T$ is strongly diagonal, $x \in[T]^{2}$ with $\operatorname{clp}(x)=\eta_{0}$ or $\operatorname{clp}(x)=\eta_{1}$ we must have $\operatorname{clp}(g[x])=\eta_{0}$ or $\operatorname{clp}(g[x])=\eta_{1}$. Similarly, for any pair $x \subseteq T$ with $\operatorname{clp}(x)=\eta_{1}$ or $\operatorname{clp}(x)=\eta_{2}$ we must have $\operatorname{clp}(g[x])=\eta_{1}$ or $\operatorname{clp}(g[x])=\eta_{2}$. Say $g$ sends $i$ to $j$ if for all incomparable pairs $x$ from $T$ with $\operatorname{clp}(x)=\eta_{i}$, one has $\operatorname{clp}(g[x])=\eta_{j}$.

Note that every element of the range of $e \circ \varphi_{w^{\prime}}$ for $w^{\prime}=\varphi_{w}(\langle 0,0\rangle)$ is an extension of $e\left(w^{\prime}\right)$ so any pair of elements of the range of $e \circ \varphi_{w^{\prime}}$ forms a short leaf triple with $e\left(\varphi_{w}(\langle 1\rangle)\right)$. Thus without loss of generality we may assume that for every pair $x_{1}, x_{2}$ from $T$ there is a node $x_{0}$ from $T$ such that $\left\{x_{0}, x_{1}, x_{2}\right\}$ is a short leaf triple with short leaf $x_{0}$ such that with $\operatorname{clp}\left(\left\{x_{0}, x_{2}\right\}\right)=\operatorname{clp}\left(\left\{x_{0}, x_{1}\right\}\right)=$ $\operatorname{clp}(\{\langle 0,0\rangle,\langle 0\rangle\})$. Then the type of $\left\{x_{0}, x_{1}, x_{2}\right\}$ depends only on the type of $\left\{x_{1}, x_{2}\right\}$. Since $d^{\prime \prime}$ is constant on triples from $T$ of the same ordered similarity type, for each $i<4, g$ sends $i$ to $j$ for some $j<4$.

Claim 5.5.c. Either $g$ sends 0 to 1 and 1 to 0 , or $g$ sends 0 to 0 and 1 to 1 .

Proof. Consider $a=\langle 0,0,0,0\rangle, b=\langle 0,1\rangle$ and $c=\langle 1,0,0\rangle$. Then $\{a, b, c\}$ is diagonal, and $c \wedge a=\emptyset=c \wedge b$.

Now $\operatorname{clp}(\{a, c\})=\eta_{1}$ and $\operatorname{clp}(\{b, c\})=\eta_{0}$. Let $s=e^{\prime}(a), t=e^{\prime}(b)$ and $u=e^{\prime}(c)$. Set $x:=\{s, u\}$ and $y=\{t, u\}$. Since $e^{\prime}$ is a polite pnp map, by Lemma 2.17, we have $\operatorname{clp}(x)=\eta_{1}$ and $\operatorname{clp}(y)=\eta_{0}$.

Since every triple of $T$ witnesses meet regularity for $g, g(u) \wedge g(s)=g(u) \wedge$ $g(t)$. That is, $g(s)$ and $g(t)$ are on the same side of $g(u)$. Let $\gamma=\lg (g(u) \wedge g(s))$. Then $g(s)(\gamma)=g(t)(\gamma)=1-g(u)(\gamma)$. If $g(s)(\gamma)=0$ then $\operatorname{clp}(g[x])=\eta_{1}$ and $\operatorname{clp}(g[y])=\eta_{0}$, so $g$ sends 1 to 1 and 0 to 0 . Otherwise $g(s)(\gamma)=1$. In this case $\operatorname{clp}(g[x])=\eta_{0}$ and $\operatorname{clp}(g[y])=\eta_{1}$, so $g$ sends 1 to 0 and 0 to 1 .

Claim 5.5.d. Either $g$ sends 2 to 3 and 3 to 2, or $g$ sends 2 to 2 and 3 to 3 . 
Proof. The proof parallels that of the previous claim using $a=\langle 1,1,1,1\rangle, b=$ $\langle 1,0\rangle$ and $c=\langle 0,0,1\rangle$. The details are left to the reader.

Claim 5.5.e. The map $\left(g \circ e^{\prime}\right) \circ\left(g \circ e^{\prime}\right)$ which preserves $<_{Q}$.

Proof. It is enough to show that for all $i<4,\left(g \circ e^{\prime}\right) \circ\left(g \circ e^{\prime}\right)$ preserves $<_{Q}$ on pairs $x, y$ whose image under $\varphi_{w}$ has type $\eta_{i}$.

Note that $e^{\prime}$ is a polite pnp map which preserves $<_{Q}$ and similarity types and takes comparable pairs to incomparable ones where $<_{Q}$ coincides with $<_{\text {lex }}$. Thus if $g$ sends 0 to 0 and 1 to 1 , then $g \circ e^{\prime}$ preserves both the order $<_{Q}$ and similarity type on pairs of type $\eta_{0}$ or $\eta_{1}$, as does its square. If $g$ sends 0 to 1 and 1 to 0 , then $g \circ e^{\prime}$ reverses order and reverses similarity type on pairs of type $\eta_{0}$ or $\eta_{1}$, so its square preserves the order $<_{Q}$ and similarity types on pairs of type $\eta_{0}$ or $\eta_{1}$.

A similar argument holds when 0 and 1 are replaced by 2 and 3 so the claim follows.

Claim 5.5.f. The maps $\left(g \circ e^{\prime}\right)$ and $\left(g \circ e^{\prime}\right) \circ\left(g \circ e^{\prime}\right)$ are pnp maps which satisfy meet regularity.

Proof. The maps are pnp maps since they are compositions of pnp maps. Now $e^{\prime}$ preserves meet regularity, and every three element subset of $T=\operatorname{ran}\left(e^{\prime}\right)$ witnesses meet regularity for $g$, so $g \circ e^{\prime}$ satisfies meet regularity as does its square.

Claim 5.5.g. For all three element subsets $x \subseteq T, \operatorname{clp}\left(g \circ e^{\prime} \circ g[x]\right)=\operatorname{clp}(x)$.

Proof. Since $e^{\prime}$ preserves $<_{Q}$ and $\left(g \circ e^{\prime}\right) \circ\left(g \circ e^{\prime}\right)$ preserves $<_{Q}$, it follows that $g \circ e^{\prime} \circ g$ preserves $<_{Q}=<_{\operatorname{lex}}$ on $T$. Since by Claim 5.5.b, every triple from $T$ witnesses meet regularity for $g$, it follows that every triple from $T$ witnesses meet regularity for $g \circ e^{\prime} \circ g$.

Recall that $g$ takes short leaf triples of $T$ to short leaf triples and preserves meet length order on such triples. Since $e^{\prime}$ preserves similarity types, $g \circ e^{\prime} \circ g$ is polite on short leaf triples $x$ from $T$ so by Lemma 2.17, $\operatorname{clp}\left(g \circ e^{\prime} \circ g[x]\right)=\operatorname{clp}(x)$.

Now suppose $x \subseteq T$ is a long leaf triple. Since $g \circ e^{\prime} \circ g$ preserves $<_{Q}$ on $T$ and has range a strongly diagonal set, by Lemma 5.3, $g \circ e^{\prime} \circ g[x]$ is a long leaf triple. Since $g \circ e^{\prime} \circ g$ also preserves meet regularity on $T, \operatorname{clp}\left(g \circ e^{\prime} \circ g[x]\right)=\operatorname{clp}(x)$ by Lemma 5.4.

Claim 5.5.h. The map $g^{\prime}:=\left(g \circ e^{\prime}\right) \circ\left(g \circ e^{\prime}\right)$ preserves meet length order.

Proof. Since $e^{\prime}$ preserves meet length order, it suffices to show that $g \circ e^{\prime} \circ g$ preserves meet length order on $z \subseteq T$ with $|z| \leq 4$. Let $z=\{x, y, u, v\} \subseteq T$ be arbitrary and assume $\lg (x \wedge y)<\lg (u \wedge v)$.

For the first case, assume $|z|=2$; let $z=\{s, t\}$ where $\lg (s)<\lg (t)$. Then there are only three possible meets, listed here in increasing order of length: $s \wedge t, s \wedge s$ and $t \wedge t$. Since $g$ is a pnp map, $\lg (g(s))<\lg (g(t))$. Since the range 
of $g$ is strongly diagonal set, $g(s)$ and $g(t)$ are incomparable, so $g(s) \wedge g(t)$ is a proper initial segment of both $g(s)$ and $g(t)$.

For the second case, assume $|z|=3$. Then $\operatorname{clp}\left(g^{\prime}[z]\right)=\operatorname{clp}(z)$ by Claim 5.5.g, so $g^{\prime}$ preserves meet regularity on $z$.

For the third case, assume $|z|=4$ and $x \wedge y \subseteq u \wedge v$. Either $(x \wedge y) \frown\langle 0\rangle \subseteq u \wedge v$ or else $(x \wedge y) \frown\langle 1\rangle \subseteq u \wedge v$. Thus either $x \wedge y=y \wedge u=y \wedge v$ or else $x \wedge y=$ $x \wedge u=y \wedge v$. Since $\operatorname{clp}\left(g^{\prime}[\{x, u, v\}]\right)=\operatorname{clp}(\{x, u, v\})$ and $\operatorname{clp}\left(g^{\prime}[\{y, u, v\}]\right)=$ $\operatorname{clp}(\{y, u, v\})$, it follows that $g^{\prime}(x) \wedge g^{\prime}(y)<g^{\prime}(u) \wedge g^{\prime}(v)$.

For the final case, assume $|z|=4$ and $x \wedge y \nsubseteq \subset \wedge \wedge$. Since $\lg (x \wedge y)<\lg (u \wedge v)$, it follows that $x \wedge y$ and $u \wedge v$ are incomparable. Thus $x \wedge v=y \wedge v \subseteq u \wedge v$. Since $z$ is strongly diagonal, so is $\{x, y, v\}$, so by Lemma $5.2, \lg \left(g^{\prime}(x) \wedge g^{\prime}(y)\right) \leq$ $\lg \left(g^{\prime}(x \wedge y)\right)$. Now $z^{\prime}=\{x \wedge y, u, v\}$ is a short leaf triple which is a subset of $z$. Since $\left(\operatorname{clp}(z), \prec_{z}\right)$ is a vip 4-type, it follows that $\left(\operatorname{clp}\left(z^{\prime}\right), \prec_{z^{\prime}}\right)$ is a vip 3-type. Thus $\operatorname{clp}\left(g^{\prime}\left[z^{\prime}\right]\right)=\operatorname{clp}\left(z^{\prime}\right)$ and $g^{\prime}\left[z^{\prime}\right]$ is a short leaf triple. In particular, $\lg \left(g^{\prime}(x \wedge\right.$ $y))<\lg \left(g^{\prime}(u) \wedge g^{\prime}(v)\right)$, hence $\lg \left(g^{\prime}(x) \wedge g^{\prime}(y)\right) \leq \lg \left(g^{\prime}(x \wedge y)\right)<\lg \left(g^{\prime}(u) \wedge g^{\prime}(v)\right)$, and $g^{\prime}$ preserves meet length order on $z$.

Since $g^{\prime}$ trivially preserves meet length order on the empty set and sets of size 1 , the claim follows from the above case analysis.

By Claims 5.5.b, 5.5.e and 5.5.h, $g^{\prime}=\left(g \circ e^{\prime}\right) \circ\left(g \circ e^{\prime}\right)$ is a polite pnp map which preserves $<_{Q}$. Therefore the lemma follows with $f=e^{\prime} \circ g \circ e^{\prime}$.

Theorem 5.6. Suppose that $\kappa$ is a cardinal which is measurable in the generic extension obtained by adding $\lambda$ Cohen subsets of $\kappa$, where $\lambda \rightarrow(\kappa)_{2^{\kappa}}^{6}$. If $g$ : $\kappa>2 \rightarrow{ }^{\kappa>} 2$ is a pnp map whose range is a strongly diagonal set whose meet closure is a subset of $S^{*}=\sigma[\kappa]$, then there is pnp map $f$ such that $g \circ f$ is a pnp diagonalization into $S^{*}$ with level harmony.

Proof. Apply Lemma 3.4 to obtain a pre- $S^{*}$-vip order $\prec$ and a sequence of pnp diagonalizations with level harmony $\left\langle\varphi_{t}: t \in{ }^{\kappa>} 2\right\rangle$ such that for all $t \in{ }^{\kappa>} 2$, the three listed properties of the lemma hold. Apply Lemma 5.5 to $g$ to obtain a pnp map $f_{0}$ such that $g \circ f_{0}$ is a polite pnp map and $f_{0}$ preserves meet regularity and meet length order on short leaf triples. Since $\psi=\varphi_{0}$ is a pnp diagonalization with level harmony, by Lemma 5.1, the map $g \circ f_{0} \circ \psi$ is a pnp diagonalization with level harmony and $f_{0} \circ \psi$ preserves meet regularity and meet length order on short leaf triples. Let $f=f_{0} \circ \psi$. Then $g \circ f$ is a pnp diagonalization with level harmony as required.

Corollary 5.7. Suppose that $\kappa$ is a cardinal which is measurable in the generic extension obtained by adding $\lambda$ Cohen subsets of $\kappa$, where $\lambda \rightarrow(\kappa)_{2^{\kappa}}^{6}$. If $g$ : $S^{*} \rightarrow{ }^{\kappa>} 2$ is a pnp map whose range is a strongly diagonal set $D$ whose meet closure is a subset of $S^{*}=\sigma[\kappa]$ and $\prec$ is a D-vip order of the levels of ${ }^{\kappa>} 2$, then every vip $\ell$-type $(\tau, \lessdot)$ for $0<\ell<\omega$ is realized as $\left(\operatorname{clp}(x), \prec_{x}\right)$ for some $x \subseteq D$ and for all $y \in[D]^{\ell},\left(\operatorname{clp}(y), \prec_{y}\right)$ is a vip $\ell$-type.

Proof. By Lemma 2.22, for all $y \in[D]^{\ell},(\operatorname{clp}(y), \prec y)$ is a vip $\ell$-type.

Use the Diagonalization Lemma 3.3 to obtain a pnp diagonalization $\varphi$ : $\kappa>2 \rightarrow S^{*}$ with level harmony. Apply Theorem 5.6 to $g \circ \varphi$ to obtain a pnp map 
$f$ so that $g \circ f$ is a pnp diagonalization with level harmony. Let $\left.D^{\prime}=g \circ f{ }^{\kappa>} 2\right]$. Since $D^{\prime} \subseteq D, D^{\prime}$ is it a strongly diagonal subset whose meet closure is a subset of $S^{*}$. Thus by Theorem 4.6 , every vip $\ell$-type $(\tau, \lessdot)$ is realized as $\left(\operatorname{clp}(x), \prec_{x}\right)$ for some $x \subseteq D^{\prime} \subseteq D$.

Definition 5.8. Suppose $\psi$ is a pnp map whose range is a strongly diagonal set $D$ and $\prec$ is a $D$-vip level order. Call $I, H \subseteq \kappa$ a coding pair for $\psi$ and $\prec$ if the following conditions are satisfied:

1. $\operatorname{clp}(u)=\operatorname{clp}(\{\langle 0,0\rangle,\langle 1\rangle\})$ for every pair $u \subseteq \psi \circ \sigma[I]$;

2. every triple $v \subseteq \psi \circ \sigma[I \cup H]$ whose intersection with $\psi \circ \sigma[I]$ is non-empty is a short leaf triple;

3. for all $\alpha \in I$ and $\beta \in H, \alpha<\beta$ and $\psi \circ \sigma(\beta)<_{\text {lex }} \psi \circ \sigma(\alpha)$.

Theorem 5.9 (Uniformization Theorem). Let $n \geq m \geq 2$ and suppose that $\kappa$ is a cardinal which is measurable in the generic extension obtained by adding $\lambda$ Cohen subsets of $\kappa$, where $\lambda \rightarrow(\kappa)_{2^{\kappa}}^{2 m^{\prime}}$ for $m^{\prime}=\max (3, m)$. There are a pnp mapping $\psi:{ }^{\kappa>} 2 \rightarrow{ }^{\kappa>} 2$ whose range is a strongly diagonal set $D$ and a D-vip level ordering $\prec$ such that for all copies $\mathbb{G}^{*}=(H, E\lceil H)$ of the Rado graph and all colorings $c$ of $[H]^{m}$ with fewer than $\kappa$ colors, there is an increasing endomorphism $h: \kappa \rightarrow H$ and $I \in[H]^{n}$ such that the following conditions hold:

1. for all positive $\ell<\omega$, every $\ell$-element subset of $\psi \circ \sigma[\kappa]$ realizes some vip $\ell$-type and every vip $\ell$-type is realized by some subset of $\psi \circ \sigma[h[\kappa]]$;

2. for all $a \in[\kappa]^{m}, \operatorname{clp}(\psi \circ \sigma[h[a]])=\operatorname{clp}(\psi \circ \sigma[a])$;

3. $c(x)=c(y)$ for all $x, y \in[I \cup h[\kappa]]^{m}$ whose images under $\psi \circ \sigma$ have the same vip m-type; and

4. $I, h[\kappa]$ is a coding pair for $\psi$ and $\prec$.

Proof. Let $\left\langle\varphi_{t}: t \in{ }^{\kappa>} 2\right\rangle$ and $\prec$ such that for all $t \in{ }^{\kappa>} 2, \varphi_{t}$ is a pnp diagonalization with level harmony into $S^{*} \cap$ Cone $(t)$; the meet closure of the set $D_{t}:=\operatorname{ran}\left(\varphi_{t}\right)$ is a subset of $S^{*}$ disjoint from $D_{s}^{\wedge}$ for all $s \prec t$; and $\prec$ is a $D_{t}$-vip order. Let $\psi=\varphi_{\emptyset}$. Then $\prec$ is a $D$-vip level order for $D=\operatorname{ran}(\psi)$.

Recall that by Lemma 2.17, if $\bar{\phi}$ is polite pnp map, then $\operatorname{clp}(\bar{\phi}[x])=\operatorname{clp}(x)$ for every strongly diagonal set $x \subseteq{ }^{\kappa>} 2$. Also, polite pnp maps are closed under composition. We use these facts repeated in this proof.

Let $\mathbb{G}^{*}=(H, E\lceil H)$ be an arbitrary copy of $\mathbb{G}$ in $\mathbb{G}$ and suppose $c$ is an arbitrary coloring of $[H]^{m}$ with fewer than $\kappa$ many colors. Apply Lemma 2.1 to get an increasing endomorphism $j: \kappa \rightarrow H$. By Lemma 2.7, $\sigma \circ j_{0} \circ \sigma^{-1}$ is a pnp map on $S^{*}$, so $(\psi \circ \sigma) \circ j_{0} \circ \psi$ is a pnp map on ${ }^{\kappa>} 2$. Let $f_{0}$ be a pnp map obtained from Theorem 5.6 such that $(p s i \circ \sigma) \circ\left(j_{0} \circ \sigma^{-1} \circ \psi \circ f_{0}\right)$ is a pnp diagonalization with level harmony. Set $g:=j_{0} \circ \sigma^{-1} \circ \psi \circ f_{0}$. Note that $\sigma \circ g$ is a pnp map.

Define a coloring $d$ on $\left[{ }^{\kappa>} 2\right]^{m}$ by $d(x)=\left(\left(\operatorname{clp}(\psi \circ \sigma[g[x]]), \prec_{\psi \circ \sigma[g[x]]}\right), c(g[x])\right)$. Apply Shelah's Theorem 2.24 to $d$ to obtain $w \in{ }^{\kappa>} 2$ and a strong embedding $e$ 
such that $e$ preserves $\prec$ on $\operatorname{Cone}(w)$ and for all $u, v \in[\operatorname{Cone}(w)]^{m},\left(\operatorname{clp}(u), \prec_{u}\right.$ )$=\left(\operatorname{clp}(v), \prec_{v}\right)$ implies $d(e[u])=d(e[v])$.

Set $K:=\left\{0^{2 i \frown}\langle 1\rangle: i<n\right\}$; let $J:=\varphi_{w} \circ \psi[K]$ and let $I:=g \circ e[J]$. Since $\psi \circ \sigma \circ g, e, \varphi_{w}$ and $\psi$ are all polite pnp maps, $\operatorname{clp}(\psi \circ \sigma[I])=\operatorname{clp}(e[J])=$ $\operatorname{clp}\left(\varphi_{w} \circ \psi[K]\right)=\operatorname{clp}(K)$.

Let $z$ be an extension of $z^{\prime}:=\varphi_{w} \circ \psi\left(0^{2 n} \frown\langle 1\rangle\right)$ such that $g \circ e(z)$ is larger than every $\alpha \in I$. Set $h:=(g \circ e) \circ\left(\varphi_{z} \circ \psi\right) \circ \sigma$. Since $\sigma \circ g$ is a pnp map, so is $\sigma \circ(g \circ e) \circ\left(\varphi_{z} \circ \psi\right)$. Thus by Lemma 2.7, $h$ is an increasing endomorphism. Since $g=j_{0} \circ \sigma^{-1} \circ \psi, h$ maps into $H$.

Claim 5.9.a. $I, h[\kappa]$ is a coding pair.

Proof. Since $J:=\varphi_{w} \circ \psi[K]$ is a subset of Cone $(w)$, the meet closure of $L:=$ $\varphi \circ \psi\left[S^{*}\right]$ is a subset of $\operatorname{Cone}(z) \subseteq \operatorname{Cone}(w)$, the only triples of $J \cup L$ which fail to be short leaf triples are subsets of $L$. Thus $I, h[\kappa]$ satisfies the second condition of the definition of coding pair,since $\psi \circ \sigma[I]=(\psi \circ \sigma) \circ(g \circ e)[J]$, $\psi \circ \sigma[h[\kappa]]=(\psi \circ \sigma) \circ(g \circ e)[L]$ and $(\psi \circ \sigma) \circ(g \circ e)$ is a polite pnp map. Similarly, $I, h[\kappa]$ satisfies condition 1 of the definition of coding pair, since clp is preserved by pnp maps and all pairs from $K$ have the desired collapse. and $I \cup h[\kappa]=$ $g \circ e\left[J \cup(\varphi \circ \psi)\left[S^{*}\right]\right]$, For all $\alpha \in I$ and $\beta \in h[\kappa]$, then the inequality $\alpha<\beta$ holds by choice of $z$ and, if $\alpha=g \circ e(u)$ and $\beta=(g \circ e)(v)$ for $v=\varphi_{w} \circ \psi\left(0^{2 i} \frown\langle 1\rangle\right)$, then the order relation $\psi \circ \sigma(\beta)<_{\operatorname{lex}} \psi \circ \sigma(\alpha)$ holds since $z^{\prime}<_{\operatorname{lex}} \varphi_{w} \circ \psi\left(0^{2 i} \frown\langle 1\rangle\right)$, $\operatorname{clp}(\{u, v\})=\operatorname{clp}\left(\left\{z^{\prime}, v\right\}\right)$, and $\psi \circ \sigma \circ g \circ e$ preserves the collapses. That is, $I, h[\kappa]$ satisfies the third condition of coding pair and hence is a coding pair.

Claim 5.9.b. for all positive $\ell<\omega$, every $\ell$-element subset of $\psi \circ \sigma[\kappa]$ realizes some vip $\ell$-type and every vip $\ell$-type is realized by some subset of $\psi \circ \sigma[h[\kappa]]$.

Proof. Since $\psi$ is a pnp diagonalization into $S^{*}$ with level harmony, and $\prec$ is a $\operatorname{ran}(\psi)$-vip level order, by Lemma 2.22 , every $\ell$-element subset realizes some vip $\ell$-type. Since $\psi \circ \sigma[h[\kappa]]$ is $(\psi \circ \sigma) \circ g \circ e \circ \varphi_{z} \circ \psi\left[S^{*}\right]$, by Corollary 5.7, every vip $\ell$-type is realized by some subset of this range.

Claim 5.9.c. For all $a \in[\kappa]^{m}, \operatorname{clp}(\psi \circ \sigma[h[a]])=\operatorname{clp}(\psi \circ \sigma[a])$.

Proof. Let $a \in[\kappa]^{m}$ be arbitrary. Since $h[a]=\left(g \circ e \circ \varphi_{z}\right)[\psi \circ \sigma[a]]$, and $(\psi \circ \sigma) \circ$ $\left(g \circ e \circ \varphi_{z}\right)$ is a polite pnp map, it follows that $\operatorname{clp}(\psi \circ \sigma[h[a]])=\operatorname{clp}(\psi \circ \sigma[a])$.

Claim 5.9.d. For all $x, y \in[I \cup h[\kappa]]^{m}$, if the images of $x$ and $y$ under $\psi \circ \sigma$ have the same vip $m$-type, then $c(x)=c(y)$.

Proof. By Corollary 5.7, every vip $m$-type is realized by some subset of the range of $\varphi_{w} \circ f \circ \psi$. Similarly, since the mapping $t \mapsto w^{\frown} t$ is a pnp map, every vip $m$-type is realized by some subset of Cone $(w)$. By choice of $e$ and $w$, for all $m$-element subsets $u, v \subseteq \operatorname{Cone}(w)$ if $\left(\operatorname{clp}(u), \prec_{u}\right)=\left(\operatorname{clp}(v), \prec_{v}\right)$, then $d(e[u])=d(e[v])$.

Thus we can define a map $\rho$ on the vip $m$-types by $\rho(\tau, \lessdot)=\left(\tau^{\prime}, \lessdot^{\prime}\right)$ is such that for all $u \subseteq \varphi_{w} \circ \psi\left[S^{*}\right]$ with $(\operatorname{clp}(u), \prec u)=(\tau, \lessdot), d(e[u])=$ 
$\left(\left(\tau^{\prime}, \lessdot^{\prime}\right), c(g[e[u]])\right)$. Since by Corollary 5.7, every vip $m$-type is realized in the range of $(\psi \circ \sigma) \circ g \circ e$, it follows that $\rho$ is surjective. Since the domain and range of $\rho$ are the same finite set, $\rho$ must also be injective, i.e. bijective.

Recall $I \cup h[\kappa]=g \circ e[J \cup L]$ for $L=\varphi_{w} \circ \psi\left[S^{*}\right]$ and $J \cup L \subseteq$ Cone $(w)$. Thus if $x$ and $y$ are arbitrary $m$-element subsets of $I \cup h[\kappa]$ whose images under $\psi \circ \sigma$ have the same vip $m$-type $\left(\tau^{\prime}, \lessdot^{\prime}\right)$, and $u$ and $v$ be subsets of Cone $(w)$ such that $x=(g \circ e)[u]$ and $y=(g \circ e)[v]$, then $u$ and $v$ must have the same vip $m$-type $(\tau, \lessdot)$ where $\rho(\tau, \lessdot)=\left(\tau^{\prime}, \lessdot^{\prime}\right)$, so $d(e[u])=d(e[v])$; it follows that $c(g \circ e[u])=c(g \circ e[v])$, that is, $c(x)=c(y)$.

Now the theorem follows from the claims.

\section{Canonical partitions and the main result}

This section is devoted to the proof of the Theorem 1.1, which, for the convenience of the reader, we restate below.

Theorem 6.1. Let $m \geq 2$ and suppose that $\kappa$ is a cardinal which is measurable in the generic extension obtained by adding $\lambda$ Cohen subsets of $\kappa$, where $\lambda \rightarrow$ $(\kappa)_{2^{\kappa}}^{2 m}$. Then for $r_{m}^{+}$equal to the number of vip m-types, any $\kappa$-Rado graph $\mathbb{G}=(\kappa, E)$ satisfies $\mathbb{G} \rightarrow(\mathbb{G})_{<\kappa, r_{m}^{+}}^{m}$ and $\mathbb{G} \nrightarrow(\mathbb{G})_{<\kappa, r_{m}^{+}-1}^{m}$. Moreover, there is a canonical partition of $[\mathbb{G}]^{m}$ into $r_{m}^{+}$parts.

Proof. Let us first note that it suffices to prove that there is a canonical partition into $r=r_{m}^{+}$parts. Namely, suppose that $\mathcal{C}=\left\{C_{0}, C_{1}, \ldots, C_{r}\right\}$ is such a partition and that $c:[\kappa] \rightarrow \mu$ for some $\mu<\kappa$ is a coloring. Then by indivisibility there is a copy $\mathbb{G}^{*}$ of $\mathbb{G}$ within $\mathbb{G}$ such that $\left[\mathbb{G}^{*}\right]^{m} \cap C_{j}$ is monochromatic for each $j<r$ and hence $\left[\mathbb{G}^{*}\right]^{m}$ is colored into at most $r$ colors. Hence $\mathbb{G} \rightarrow(\mathbb{G})_{<\kappa, r_{m}^{+}}^{m}$. On the other hand, if we define a coloring $c$ of $[\kappa]^{m}$ by letting $c(z)=j$ iff $z \in C_{j}$ we obtain a coloring into $<\kappa$ colors in which every copy $\mathbb{G}^{*}$ of $\mathbb{G}$ within $\mathbb{G}$ has all $r_{m}^{+}$-colors, by indivisibility. Therefore $\mathbb{G} \nrightarrow(\mathbb{G})_{<\kappa, r_{m}^{+}-1}^{m}$.

Let us prove the existence of a canonical partition. Apply Theorem 5.9 to to obtain a pnp mapping $\psi$ whose range is a strongly diagonal set $D$ and a $D$-vip level order $\prec$ with the specified properties.

With $r=r_{m}^{+}$, let $\left(\tau_{0}, \lessdot_{0}\right),\left(\tau_{1}, \lessdot_{1}\right), \ldots,\left(\tau_{r-1}, \lessdot_{r-1}\right)$ enumerate the vip $m$ types. For $j<r$, let $C_{j}$ be the set of all $m$-element subsets $A \subseteq \kappa$ for which $\left(\operatorname{clp}(\psi \circ \sigma[A]), \prec_{\psi \circ \sigma[A]}\right)=\left(\tau_{j}, \lessdot_{j}\right)$. Then $\mathcal{C}:=\left\{C_{0}, C_{1}, \ldots, C_{r-1}\right\}$ is a partition of $[\kappa]^{m}$ into $r$ sets.

Claim 6.1.a. $\mathcal{C}$ is persistent.

Proof. Suppose that $\mathbb{G}^{*}=(H, E\lceil H)$ is an isomorphic copy of the $\kappa$-Rado graph within $\mathbb{G}$.

If $m=2$, apply Lemma 2.1 to get an increasing map $h: \kappa \rightarrow H$, observe that $\sigma^{-1} \circ h \circ \sigma$ is a pnp map by the Translation Theorem 2.8, and $\psi \circ h \circ \sigma$ is a pnp map whose range is a strongly diagonal subset of ${ }^{\kappa>}$ 2. By Theorem 4.1 
of [3], there is a similarity embedding of every 2-type into the range of $\psi \circ h \circ \sigma$, i.e. every 2-type can be realized by some subset of $\psi \circ \sigma[h[\kappa]]$. By Lemma 2.22, every equivalence class of the partition has non-empty intersection with $[H]^{2}$.

Now suppose $m>2$. Let $c$ be the coloring defined on $x \in[H]^{m}$ by $c(x)=$ $\left(\operatorname{clp}(\psi \circ \sigma[x]), \prec_{\psi \circ \sigma[x]}\right)$. Since the range of $\psi$ is a strongly diagonal set $D$ and $\prec$ is a $D$-vip level order, $c$ maps into the set of all vip $m$-types, which is finite. Let $h: \kappa \rightarrow H$ be the increasing endomorphism whose existence is guaranteed by the Uniformization Theorem 5.9. By the first condition $h$ must satisfy, every vip $m$-type is realized by a subset of $\operatorname{ran}(h) \subseteq H$, so every equivalence class meets $[H]^{m}$ and $\mathcal{C}$ is persistent.

Claim 6.1.b. For all copies $\mathbb{G}^{*}$ of $\mathbb{G}$, the restriction of the partition $\mathcal{C}$ to $m$ element subsets of $\mathbb{G}^{*}$ is indivisible.

Proof. Fix a copy $\mathbb{G}^{*}=\left(H, E\lceil H)\right.$ of $\mathbb{G}$ and suppose $c$ is a coloring of $[H]^{m}$ with $\mu<\kappa$ many colors.

If $m=2$, let $h$ be as in argument for $m=2$ in the previous claim, apply Shelah's Theorem to $d(x):=(\operatorname{clp}(\psi \circ \sigma[g[x]], c(g[x]))$ for $g=h \circ \sigma$ to get $e$ and $w$ so that for $x \subseteq$ Cone $(w), d(x)$ depends only on the ordered similarity type of $x$, hence only on the similarity type of $x$. Continue as in Claim 5.9d.

So suppose $m>2$. Let $h: \kappa \rightarrow H$ be the increasing endomorphism whose existence is guaranteed by the Uniformization Theorem 5.9. By the third condition $h$ must satisfy, $c$ is monochromatic on $m$-element subsets of $h[\kappa]$ of the same vip $m$-type. Hence $\mathbb{G}^{* *}=\left(h[\kappa], E\lceil h[\kappa])\right.$ is a copy of $\mathbb{G}$ in $\mathbb{G}^{*}$ such that $c$ is monochromatic on $[h[\kappa]]^{m} \cap C_{j}$ for each $j<r$. Therefore $\mathcal{C}$ is indivisible on $\mathbb{G}^{*}$ and the claim follows.

By the claims, $\mathcal{C}$ is a canonical partition and the theorem follows.

Definition 6.2. A family $\mathfrak{B}$ forms a basis for the equivalence relations on the $n$-element subsets of $\mathbb{G}$ if the following two conditions hold:

1. For every equivalence relation $F$ on the $[\kappa]^{n}$ and every copy $\mathbb{G}^{*}$ of $\mathbb{G}$, there is a copy $\mathbb{G}^{* *}$ of $\mathbb{G}$ in $\mathbb{G}^{*}$ and an element $B \in \mathfrak{B}(n)$ such that $F \uparrow\left[\mathbb{G}^{* *}\right]^{n}=B \uparrow\left[\mathbb{G}^{* *}\right]^{n}$.

2. If $F_{1}$ and $F_{2}$ are different elements of $\mathfrak{B}(n)$ and $\mathbb{G}^{*}$ is a copy of $\mathbb{G}$ in $\mathbb{G}$, then $F_{1} \uparrow\left[\mathbb{G}^{*}\right]^{n} \neq F_{2} \uparrow\left[\mathbb{G}^{*}\right]^{n}$.

Using results of this paper and Shelah's Theorem, Larson and Mitchell in a forth-coming paper prove there is a finite basis for the for the equivalence relations on the $m$-element subsets of $\mathbb{G}$.

We finish with some ideas on the size of $r_{m}^{+}$. There are four 2-types and each of them admits a single vip order. Any copy of an uncountable Rado $\mathbb{G}$ has an induced subgraph which is a countable Rado graph. Since Laflamme, Sauer and Vuksanovic [3] have shown that these four types must appear in translations of every induced subgraph of the countable Rado graph which is itself isomorphic to the countable Rado graph, it follows from their work that $\mathbb{G}_{\kappa} \nrightarrow\left(\mathbb{G}_{\kappa}\right)_{<\omega, r_{2}^{+}-1}^{2}$. 


\begin{tabular}{|r|r|r|}
\hline$m$ & $r_{m}^{+}$ & $r_{m}$ \\
\hline 1 & 1 & 1 \\
\hline 2 & 4 & 4 \\
\hline 3 & 128 & 112 \\
\hline 4 & 26,368 & 12,352 \\
\hline 5 & $41,932,288$ & $4,437,760$ \\
\hline
\end{tabular}

Figure 1: Some small values of $r_{m}^{+}$and $r_{m}$.

For larger values of $m$, we use Shelah's Theorem. Since the partition we use in the proof of Theorem 1.1 is definable without Shelah's Theorem we focus attention on the following question.

Question 6.3. Suppose $\kappa$ is an uncountable cardinal with $\kappa^{<\kappa}=\kappa, \mathbb{G}_{\kappa}$ is a $\kappa$-Rado graph and $2<m<\omega$. Does $\mathbb{G} \nrightarrow(\mathbb{G})_{<\omega, r_{m}^{+}-1}^{m}$ ? i.e., does the lower bound hold even when $\kappa$ does not satisfy the hypothesis of Shelah's Theorem?

Figure 1 summarizes the calculation from [4] of values of $r_{m}^{+}$for $m \leq 5$. A comparison with $r_{m}$, the number of $m$-types, is also included, where $r_{m}$ is the critical value for finite colorings of $m$-tuples of the countable Rado graph.

\section{References}

[1] Mirna Džamonja, Jean A. Larson, and William J. Mitchell. A partition theorem for a large dense linear order. To appear in the Israel Journal of Mathematics.

[2] Paul Erdős, Andras Hajnal, and Lajos Pósa. Strong embeddings of graphs into colored graphs. In Infinite and Finite Sets, Vol. I (Colloq., Keszthely, 1973; dedicated to P. Erdös on his 60th birthday), volume 10 of Colloquia Mathematica Societatis Janós Bolyai, pages 585-595. North-Holland, Amsterdam, 1975.

[3] Claude Laflamme, Norbert W. Sauer, and Vojkan Vuksanovic. Canonical partitions of universal structures. Combinatorica, 26(2):183-205, 2006.

[4] Jean A. Larson. Graph coding trees and level orders. Preprint.

[5] Jean A. Larson. Ramsey theory for countable binary homogeneous structures. Notre Dame Journal for Formal Logic, 46(3):335-352, 2005.

[6] Saharon Shelah. Strong partition relations below the power set: consistency - was Sierpinski right? II. In Sets, Graphs and Numbers (Budapest, 1991), Colloq. Math. Soc. János Bolyai, Vol. 60, pages 637-688. North-Holland, Amsterdam, 1991. Sh: 288. 